\title{
Stability Conditions for Affine Type A
}

\section{P. J. Apruzzese ${ }^{1} \cdot$ Kiyoshi Igusa ${ }^{2}$}

Received: 28 December 2018 / Accepted: 20 September 2019 / Published online: 8 November 2019

(C) The Author(s) 2019

\begin{abstract}
We construct maximal green sequences of maximal length for any affine quiver of type $A$. We determine which sets of modules (equivalently $c$-vectors) can occur in such sequences and, among these, which are given by a linear stability condition (also called a central charge). There is always at least one such maximal set which is linear. The proofs use representation theory and three kinds of diagrams shown in Fig. 1. Background material is reviewed with details presented in two separate papers Igusa $(2017 \mathrm{a}, \mathrm{b})$.
\end{abstract}

Keywords Maximal green sequences · Cluster mutation · Quivers · c-vectors · Central charge $\cdot$ Wire diagram $\cdot$ Wall crossing

Mathematics Subject Classification (2010) 16G20

\section{Introduction}

Maximal green sequences are one incarnation of a variety of related concepts of current interest. The term was defined by Keller [13] who showed that maximal green sequences can be used to compute the Donalson-Thomas invariant of a quiver. Much of the literature is devoted to the existence of such sequence. [9, 21, 27]. For example, [22] shows that the existence of a maximal green sequence, or even a weaker version known as a "reddening sequence" gives a canonical basis for the upper cluster algebra. However, this paper deals with the case of an acyclic quiver where a maximal green sequence always exists.

One important class of maximal green sequences come from stability conditions, a concept introduced much earlier by King [20]. This was extended to the study of vector bundles over projective space by Rudakov [26] who showed that a stability condition gives a Harder-

Presented by: Henning Krause.

Kiyoshi Igusa

igusa@brandeis.edu

P. J. Apruzzese

paul.apruzzese@uconn.edu

1 Department of Mathematics, University of Connecticut, Storrs, CT 06269, USA

2 Department of Mathematics, Brandeis University, Waltham, MA 02454, USA 
Narasimhan (HN)-filtration for a vector bundle. Bridgeland [3, 4] extended these definitions and results to more general triangulated categories. The connection between these concepts comes from the fact that the dimension vectors of stable modules, in order of their "slopes" forms a maximal green sequence. It is shown in [16] that an HN-stratification of the module category of any finite dimensional algebra is equivalence to a notion called a "forward hom-orthogonal sequence of bricks". In [13] this is shown to be equivalent to a maximal chain in the poset of functorially finite torsion classes.

Although maximal green sequences were defined for cluster algebras and cluster-tilted algebras as certain sequences of mutations of clusters in the cluster category $[7,8]$, the definition can be extended to arbitrary finite dimensional algebras, namely, they can be defined as maximal (finite) chains in the poset of functorially finite torsion classes or as finite wall-crossing sequences in the semi-invariant picture (or scattering diagram) of the algebra. (See [6].) The wall-crossing description is one of the techniques we use in this paper. (See Fig. 1 (1) below.) One task that is not completely finished is to show that the various definitions are equivalence in situation where more than one of them make sense.

More detailed background on the relationship between maximal green sequence, maximal chains of functorially finite torsion classes, Harder-Narasimhan filtrations, Bridgeland stability conditions, forward hom-orthogonal sequences of Schurian modules (also called bricks) and wall-crossing sequences can be found in [6, 14, 16, 17].

We investigate the lengths of maximal green sequences. By the "no gap conjecture" of [5] proved in [15] for tame quivers, the set of such lengths forms an interval. The minimum length of a maximal green sequence for cluster tilted algebras of type $A_{n}$ is given in [25]. The maximum length for the same algebras is given in many cases in [17]. In this paper we give the maximum length of a maximal green sequence for a quiver of type $\widetilde{A}_{a, b}$. (Theorem M1.) For an acyclic quiver the minimum length of a maximal green sequence is always equal to the number of vertices of the quiver.

The main topic addressed in this paper is the question of linearity of maximal green sequences of maximum length. (Definition 4.9) This question originates in a conjecture by Reineke [24] in which he asks for a linear stability condition on a Dynkin quiver which makes all indecomposable modules stable. Isomorphism classes of such modules are in bijection with the positive roots of the underlying root system. Reineke showed that the sequence of stable positive roots corresponding to the stable modules of a linear stability condition gives a quantum dilogarithm identity and he wanted that identity to have one term for every positive root. Qiu [23] has shown that, for every Dynkin quiver, there exists an orientation of the quiver and a linear stability condition given by a central charge which makes all indecomposable modules stable. This had already been done in type $A_{n}$ with straight orientation by Reineke [24]. So, [23] dealt with quivers of other Dynkin types.

It is very easy to see that there are nonlinear stability conditions (equivalent to maximal green sequences) which make all positive roots stable, namely take all indecomposable modules going, depending on sign convention, either from left to right (Reineke's sign convention) or from right to left (our sign convention) in the Auslander-Reiten quiver of the path algebra. More precisely, we order the indecomposable modules in such a way that, for every irreducible map $A \rightarrow B, B$ comes before $A$. Thus the question is mainly about the linearity of the stability condition.

In this paper we prove Reineke's original conjecture in type $A_{n}$ with any orientation and we give a complete resolution to the extension of this question to quivers of type $\widetilde{A}_{n-1}$. Since there are infinitely many positive roots in that case, the corresponding problem is to find maximal green sequences of maximum finite length and to determine which are linear. Our results are the following. 
Recall that $\widetilde{A}_{a, b}$, for positive integers $a, b$, denotes a cyclic quiver with $a$ arrow going clockwise and $b$ arrows going counterclockwise. For example, there are, up to isomorphism, two quivers of type $\widetilde{A}_{3,2}$ which we denote: (See (4.2) for the sign notation.)
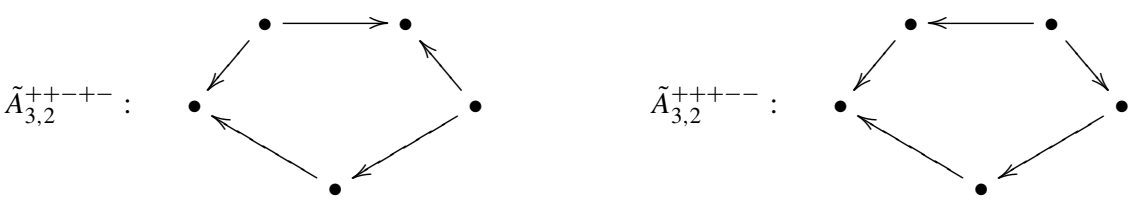

Theorem M1 Maximal green sequences for any quiver of type $\widetilde{A}_{a, b}$ have maximum length

$$
\left(\begin{array}{c}
a+b \\
2
\end{array}\right)+a b
$$

Theorem M1 is shown in two steps: In Theorem 7.10 we construct maximal green sequences of this length and in Theorem 6.11 we show that there are no maximal green sequences of greater length. Theorem M1 is already known in the case $b=1$ by [19].

Although maximal green sequences were originally defined combinatorially [13], we use the language of representation theory which we review in Section 2.1. We take a fixed field $K$ and, for any acyclic quiver $Q$, we take the path algebra $\Lambda=K Q$ of $Q$. We use the "wall-crossing" definition of a maximal green sequence from $[6,16]$ which we review in Section 4. A maximal green sequence for a finite dimensional algebra $\Lambda$ is a finite sequence of indecomposable modules $M_{1}, \cdots, M_{m}$ for which there exists a "green path" $\gamma$ going through the walls $D\left(M_{1}\right), \cdots, D\left(M_{m}\right)$ in that order and no other walls. The set of modules $M_{i}$ in this sequence are called the stable modules of the sequence.

Maximal green sequences for $Q$ are in bijection with those for $K Q$ given by the wall crossing definition in Section 4 and the dimension vectors of the stable module $M_{i}$ are the $c$-vectors of the corresponding combinatorially defined maximal green sequence. (See [16]).

For quivers of Dynkin type, the longest green path passes through all of the walls and all indecomposable modules are stable. For quivers of type $\widetilde{A}_{a, b}$ there are infinitely many indecomposable modules and each maximal green sequence makes only finitely many of them stable. In this paper we determine all possible sets of stable modules of the maximum size given in Theorem M1 above. This is summarized by the following two theorems.

Theorem M2 For every quiver of type $\widetilde{A}_{a, b}$ with $(a, b) \neq(2,2)$ there are exactly $a b$ possible sets of stable modules for the maximal green sequences of length $\left(\begin{array}{c}a+b \\ 2\end{array}\right)+a b$.

These sets are denoted $\mathcal{S}_{k \ell}$ where $\varepsilon_{k}=+, \varepsilon_{\ell}=-$ and $0<k<\ell<k+n \leq 2 n$. See Definition 6.10. For $(a, b) \neq(2,2)$ these sets are distinct. (Theorem 6.13, Corollary 7.11.) Since there are $a$ choices for $k$ and $b$ choices for $\ell$, there are $a b$ such sets. For $(a, b)=(2,2)$ the four sets $\mathcal{S}_{k \ell}$ are not distinct by Proposition 6.14 and we obtain the following.

Theorem M3 For the quivers $\widetilde{A}_{2,2}^{+-+-}$, resp. $\widetilde{A}_{2,2}^{++-}$, there are 2 , resp. 3 , distinct sets of stable modules for the maximal green sequences of the maximum length which is 10.

Returning to the linearity question, we prove first that Reineke's conjecture holds for quivers of type $A_{n}$ with any orientation. 
Theorem L1 (Corollary 2.9) For a quiver of type $A_{n}$ with any orientation, there exists a standard linear stability condition making all indecomposable modules stable.

In the affine case $\widetilde{A}_{a, b}$, we show that the following analogue of Reineke's conjecture hold.

Theorem L2 For any quiver of type $\widetilde{A}_{a, b}$ there exists a linear stability condition for which the number of stable modules is $\left(\begin{array}{c}a+b \\ 2\end{array}\right)+a b$, the maximum possible by Theorem M1.

Theorem 7.6 describes which of the sets $\mathcal{S}_{k \ell}$ are linear, i.e., realized by linear stability conditions. For example, $\mathcal{S}_{k \ell}$ is linear when $|k-\ell| \leq 2$. Theorem 7.6 implies the following.

Theorem L3 For the quiver $\widetilde{A}_{a, b}^{\varepsilon}$, if either a or $b$ is $\leq 2$, every possible set of stable modules of the maximum size is linear. Otherwise (when $a, b \geq 3$ ) there is at least one orientation of the quiver (choice of $\varepsilon$ ) for which one of the sets of stable modules of the maximum size is not linear.

The smallest nonlinear example is $\widetilde{A}_{3,3}^{++---}$where 1 of the $a b=9$ sets of stable modules is nonlinear. And, in fact, this example is the cause of all nonlinearity. For every nonlinear example, +++--- will be a subsequence of the sign pattern $\varepsilon$ up to cyclic order.

By the Deletion Lemma 6.15, maximal green sequences for $\widetilde{A}_{a, b}^{\varepsilon}$ restrict to maximal green sequences for $\widetilde{A}_{a^{\prime}, b^{\prime}}^{\varepsilon^{\prime}}$ for any subsequence $\varepsilon^{\prime}$ of $\varepsilon$ (in particular, $a^{\prime} \leq a, b^{\prime} \leq b$ ). By Remark 6.17, this includes the exceptional case where $b=0$. Although " $\widetilde{A}_{n, 0}$ ", being an oriented cycle, has an infinite-dimensional path algebra, only modules of length $\leq n$ occur in a maximal green sequence since longer modules are not "bricks" (See Proposition 4.5). In this paper we also exclude modules of length $n$. Equivalently, we mod out $\mathrm{rad}^{n-1}$, and we have one of the well-known cluster-tilted algebras of type $D_{n}$ for $n \geq 4$ considered in [17]. In Theorem 6.18 and Corollary 6.19 we show the following.

Corollary L4 For $\Lambda_{n}=K Q_{n} /$ rad $^{n-1}$ the path algebra of the oriented $n$-cycle $Q_{n}$ modulo the relation $\mathrm{rad}^{n-1}=0$, the longest maximal green sequence has length $\left(\begin{array}{c}n \\ 2\end{array}\right)+n-1$, there are $n$ different sets of stable modules of this size and all of them are linear.

In order to prove these theorem we use the following three types of diagrams where (2) and (3) are always planar. (Fig. 1 gives an example.)

(1) Wall crossing diagrams. $M$ is stable when a green path $\gamma: \mathbb{R} \rightarrow \mathbb{R}^{n}$ passes through the interior of the wall $D(M)$. The stability condition given by $\gamma$ is linear when $\gamma$ is a straight line.

(2) Chords in the "stability polygon". Certain chords represent stable modules in a linear stability condition.

(3) Wire diagrams. Stable modules are indicated by certain crossings of wires in the plane. When the wires are straight lines, this is a linear stability condition.

In Section 3 we use wall crossing diagrams to prove basic theorems about maximal green sequences, linear and nonlinear. Wire diagrams, introduced in Sections 2 and 4, are used in Section 5 to show that $\left(\begin{array}{c}a+b \\ 2\end{array}\right)+a b$ is an upper bound for the maximum length of a maximal 

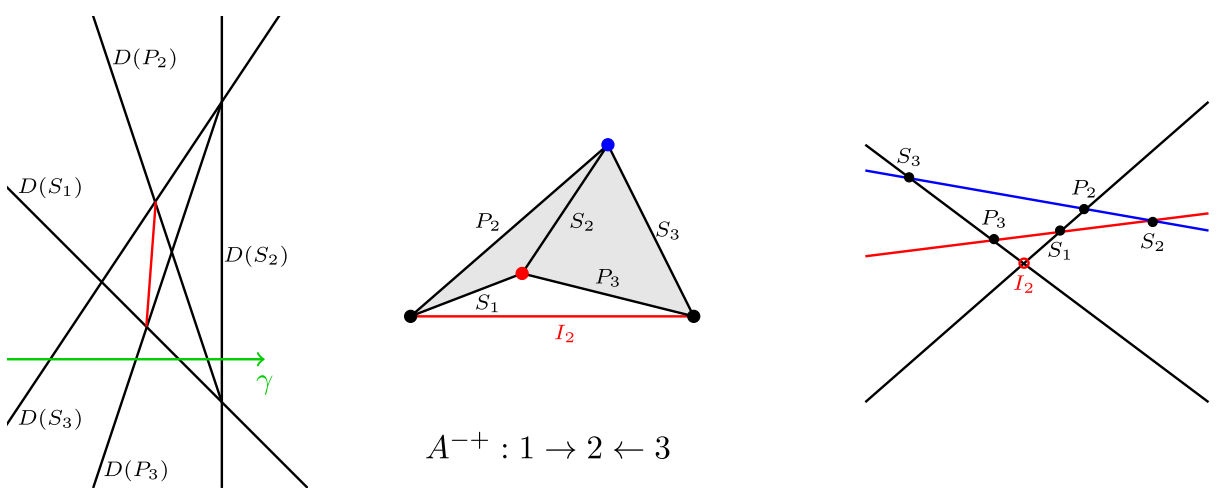

Fig. 1 Three diagrams for the same linear stability condition on a quiver of type $A_{3}$ with stable modules $S_{3}, P_{3}, S_{1}, P_{2}, S_{2}$. Each diagram shows $I_{2}$ to be unstable: (1) $\gamma$ does not pass through the wall $D\left(I_{2}\right)$ (in red), (2) the chord $I_{2}$ is outside the polygon and (3) the vertex $I_{2}$ has a red line above it

green sequence on any quiver of type $\tilde{A}_{a, b}$. Finally, in Section 6, chord diagrams, introduced in Section 1, are used to realize this upper bound.

\section{Chord Diagrams for $A_{n}$}

In this section we will review the representation theory of quivers. Details can be found in $[1,2]$. We also give the precise statement of Reineke's conjecture [24] and a proof of the conjecture in type $A_{n}$ using chord diagrams.

\subsection{Representations of Quivers}

Suppose that $Q$ is a quiver, i.e., a finite oriented graph, with vertex set $Q_{0}=\{1,2, \cdots, n\}$, arrow set $Q_{1}$ and no oriented cycles. An important case is when $Q$ is a linear quiver, in which case we say it has type $A_{n}$. For example,

$$
Q: 1 \stackrel{\alpha}{\rightarrow} 2 \stackrel{\beta}{\rightarrow} 3 \stackrel{\gamma}{\leftarrow} 4
$$

is a quiver of type $A_{4}$.

There are $2^{n-1}$ possible orientations for the arrows in a quiver of type $A_{n}$. We specify the orientation with a sign function which we define to be any mapping

$$
\varepsilon:[0, n]=\{0,1,2, \cdots, n\} \rightarrow\{-, 0,+\}
$$

written $\varepsilon(i)=\varepsilon_{i}$, so that $\varepsilon_{i}=0$ iff $i=0$ or $n$. In the corresponding linear quiver, denoted $A_{n}^{\varepsilon}$, the $i$ th arrow points left $i \leftarrow i+1$ when $\varepsilon_{i}=+$ and right $i \rightarrow i+1$ when $\varepsilon_{i}=-$. Thus, the example above is $A_{4}^{--+}$where we drop the values $v_{0}=v_{n}=0$ from the notation.

A representation $M$ of a quiver $Q$ over a field $K$ is defined to be a sequence of finite dimensional vector spaces $M_{i}, i=1, \cdots, n$ and linear maps $M_{a}: M_{i} \rightarrow M_{j}$ for every arrow $a: i \rightarrow j$ in $Q$. The dimension vector of $M$ is

$$
\underline{\operatorname{dim}} M:=\left(\operatorname{dim}_{K} M_{1}, \cdots, \operatorname{dim}_{K} M_{n}\right) .
$$


The dimension of $M$ is the dot product

$$
\operatorname{dim}_{K} M:=\sum \operatorname{dim}_{K} M_{i}=(1,1, \cdots, 1) \cdot \underline{\operatorname{dim}} M .
$$

Recall that the positive roots of the Dynkin diagram $A_{n}$ are the integer vectors

$$
\beta_{i j}:=e_{i+1}+e_{i+2}+\cdots+e_{j}=(0, \cdots, 0,1,1, \cdots, 1,0, \cdots, 0)
$$

with $1 \mathrm{~s}$ in positions $i+1, i+2, \cdots, j$ for any $0 \leq i<j \leq n$. These are the dimension vectors of the indecomposable representations of $A_{n}^{\varepsilon}$ for any $\varepsilon$.

A representation of an acyclic quiver $Q$ is equivalent to a finitely generated module over the path algebra $\Lambda=K Q$. See, e.g., [1]. A representation is indecomposable if it is indecomposable as a $\Lambda$-module. We say $N$ is a subrepresentation or submodule of $M$ if, considered as $\Lambda$-modules, $N$ is a submodule of the module $M$. Equivalently, $N_{i} \subseteq M_{i}$ for all $i \in Q_{0}$ and $M_{a}\left(N_{i}\right) \subseteq N_{j}$ for all $a: i \rightarrow j$ in $Q_{1}$.

The following proposition is an easy exercise.

Proposition 2.1 As representations of the quiver $A_{n}^{\varepsilon}, M_{i j}$ is a subrepresentation of $M_{p q}$ if and only if the following three conditions are satisfied.

(1) $p \leq i<j \leq q$

(2) Either $p=i$ or $\varepsilon_{i}=-$

(3) Either $j=q$ or $\varepsilon_{j}=+$

\subsection{Linear Stability Conditions}

We consider the dimension vectors of $\Lambda$-modules to be elements of $K_{0} \Lambda=\mathbb{Z}^{n}$. A central charge on $\Lambda=K Q$ is defined to be an additive mapping

$$
Z: K_{0} \Lambda \rightarrow \mathbb{C}
$$

of the form

$$
Z(x)=\mathbf{a} \cdot x+i \mathbf{b} \cdot x=r(x) e^{i \theta(x)}
$$

for fixed vectors $\mathbf{a}, \mathbf{b} \in \mathbb{R}^{n}$ so that every coordinate $b_{i}$ of $\mathbf{b}$ is positive. We say that $Z$ is standard if $\mathbf{b}=(1,1, \cdots, 1)$. We also say that $\mathbf{b}$ is standard in that case.

For a $\Lambda$-module $M$, the slope of $M$ is then defined to be

$$
\sigma_{Z}(M):=\frac{\mathbf{a} \cdot \underline{\operatorname{dim}} M}{\mathbf{b} \cdot \underline{\operatorname{dim}} M}=\cot \theta(M) .
$$

In the standard case, $\mathbf{b} \cdot \underline{\operatorname{dim}} M=\operatorname{dim}_{K} M$ and the slope of the simple module $S_{i}$ is $\sigma_{Z}\left(S_{i}\right)=$ $a_{i} / b_{i}=a_{i}$. The slope is undefined for $M=0$.

Definition 2.2 [3] A $\Lambda$-module $M$ is called $Z$-semistable if

$$
\sigma_{Z}\left(M^{\prime}\right) \geq \sigma_{Z}(M)
$$

(equivalently, $\theta\left(M^{\prime}\right) \leq \theta(M)$ ) for all nonzero submodules $M^{\prime} \subseteq M$. $M$ is called $Z$-stable if every proper submodule $M^{\prime} \subset M$ has strictly larger slope: $\sigma_{Z}\left(M^{\prime}\right)>\sigma_{Z}(M)$.

Because of this definition we often refer to $Z$ as a linear stability condition. The problem is to determine the maximum finite number of stable modules given by a linear stability condition. It is an easy exercise to show that any $Z$-stable module is indecomposable.

Reineke's original conjecture states: 
Conjecture 2.3 [24] For $Q$ a Dynkin quiver, there exist a standard linear stability condition making all indecomposable modules stable.

Lutz Hille has claimed, privately to the second author, that this conjecture is not true in type $E_{6}$. Yu Qiu has shown [23] that this conjecture holds for at least one orientation of each Dynkin diagram if we drop the restriction that the linear stability condition should be standard. We will prove the original conjecture for $A_{n}^{\varepsilon}$ for any sign function $\varepsilon$ using chord diagrams.

\subsection{Chord Diagrams for Type $A_{n}$}

For a central charge $Z: K_{0}\left(K A_{n}^{\varepsilon}\right) \rightarrow \mathbb{C}$ given by $Z(x)=\mathbf{a} \cdot x+i \mathbf{b} \cdot x$, we will construct a "stability polygon" $C(Z) \subseteq \mathbb{R}^{2}$ which will visually display which roots $\beta_{i j}$ are stable. This "polygon" might be degenerate, i.e., one-dimensional. by

The vertices of the stability polygon (also called dual vertices of the quiver) are given

$$
p_{i}=\left(x_{i}, y_{i}\right):=\left(b_{1}+\cdots+b_{i}, a_{1}+\cdots+a_{i}\right)
$$

for $i=0, \cdots, n$. In particular $p_{0}=(0,0)$. For standard $Z, x_{i}=i$ for all $i$. The sign of $p_{i}$ is $\varepsilon_{i}$ and we sometimes write $p_{k}^{+}$(or $p_{\ell}^{-}$) to mean $p_{k}$ which has sign $\varepsilon_{k}=+$ (or: $p_{\ell}$ with $\left.\varepsilon_{\ell}=-\right)$. We say that the vertex $p_{k}$ is positive, resp negative, if $\varepsilon_{k}=+$, resp $\varepsilon_{k}=-$. Nonnegative means either positive of equal to $p_{0}$ or $p_{n}$ which have sign 0 . Nonpositive is similarly defined.

Lemma 2.4 The slope of the line segment $V_{i j}:=\overline{p_{i} p_{j}}$ is equal to the slope of $M_{i j}$ :

$$
\text { slope } V_{i j}=\frac{a_{i+1}+\cdots+a_{j}}{b_{i+1}+\cdots+b_{j}}=\frac{\boldsymbol{a} \cdot \beta_{i j}}{\boldsymbol{b} \cdot \beta_{i j}}=\sigma_{Z}\left(M_{i j}\right) \text {. }
$$

The line segments $V_{i j}$ will be called chords of the stability polygon $C(Z)$ defined below.

In the sequel we will use the words "above" and "below" to refer to relative position in the plane. Thus, a point $\left(x_{0}, y_{0}\right)$ is above, resp. below, a subset $S \subseteq \mathbb{R}^{2}$ if $S$ contains a point $\left(x_{0}, z\right)$ with $y_{0}>z$, resp. $y_{0}<z$. We use "higher" and "lower" when referring only to the difference in the $y$-coordinates.

Theorem 2.5 The module $M_{i j}$ is Z-semistable if and only if the following conditions hold.

(1) For all $i<k<j$ with $\varepsilon_{k}=+$, the point $p_{k}^{+}$lies on or above the chord $V_{i j}$.

(2) For all $i<\ell<j$ with $\varepsilon_{\ell}=-$, the point $p_{\ell}^{-}$lies on or below the chord $V_{i j}$.

$M_{i j}$ is $Z$-stable if and only if it is Z-semistable and $p_{i}, p_{j}$ are the only vertices on $V_{i j}$.

Proof We discuss only the stable case. The semistable case is similar.

$(\Rightarrow) \quad$ Suppose $M_{i j}$ is $Z$-stable. Since $M_{i k}$ and $M_{\ell j}$ are submodules of $M_{i j}$ for $\varepsilon_{k}=$ ,$+ \varepsilon_{\ell}=-$ the slopes of the chords $V_{i k}, V_{\ell j}$ must be greater than the slope of $V_{i j}$. This holds if and only if $p_{k}$ is above $V_{i j}$ and $p_{\ell}$ is below $V_{i j}$. Thus (1) and (2) hold.

$(\Leftarrow) \quad$ Given (1) and (2), we have seen that submodules of $M_{i j}$ of the form $M_{i k}, M_{\ell j}$ will have slope greater than the slope of $M_{i j}$. The remaining indecomposable submodules are $M_{\ell k}$. But $V_{\ell k}$ has slope greater than that of $V_{i j}$ since it starts at the point $p_{\ell}^{-}$below $V_{i j}$ and ends at $p_{k}^{+}$above $V_{i j}$. Thus $M_{i j}$ is $Z$-stable. (See Fig. 2.) 
Fig. 2 The chords $V_{i k}, V_{\ell k}$ and $V_{\ell j}$ have slope greater than that of $V_{i j}$ if $p_{k}^{+}$is above and $p_{\ell}^{-}$is below the chord $V_{i j}$

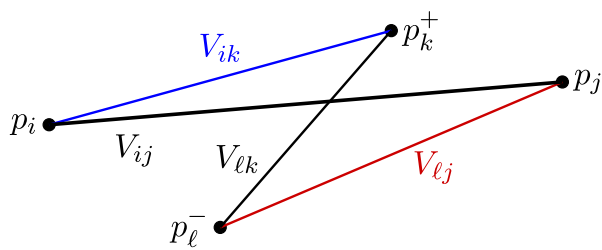

We will reformulate this theorem in terms of a polygon $C(Z)$ whose vertices are the points $p_{i}, i=0, \cdots, n$. The main property of $C(Z)$ will be that $M_{i j}$ is $Z$-semistable if and only if $V_{i j} \subseteq C(Z)$. We define the stability polygon $C(Z)$ to be the intersection $C(Z)=C^{+}(Z) \cap C^{-}(Z)$ where $C^{+}(Z), C^{-}(Z)$ are defined below. Figure 3 gives an example.

Let $P^{+}=\left\{p_{k}: \varepsilon_{k} \geq 0\right\}$, be the set of all nonnegative vertices. Number the elements of $P^{+}$as $p_{k_{i}}$ where $0=k_{0}<k_{1}<\cdots<k_{m}=n$. In Fig. 3, these are $k_{i}=0,1,3,8$. For every pair of consecutive elements $p_{k_{i-1}}, p_{k_{i}}$, let $C_{i}^{+}(Z)$ be union of the convex hull of the points $p_{j}$ for all $k_{i-1} \leq j \leq k_{i}$ with the set of all points below this convex hull. Let $C^{+}(Z)=\bigcup C_{i}^{+}(Z)$ be the union of these sets. In the example in Fig. $3, C^{+}(Z)$ is the union of three sets: $C_{1}^{+}(Z)$ is the chord $V_{01}$ and points below, $C_{2}^{+}(Z)$ is the chord $V_{13}$ and points below and $C_{3}^{+}(Z)$ is the union of the two chords $V_{34}$ and $V_{48}$ and the points below these two chords, i.e., $C^{+}(Z)$ is the blue curve and everything below the blue curve.

Lemma 2.6 For any $0 \leq i<j \leq n$, the chord $V_{i j}$ lies in $C^{+}(Z)$ if and only if $p_{k}^{+}$lies on or above $V_{i j}$ for all positive vertices $p_{k}^{+}$between $p_{i}$ and $p_{j}$, i.e., so that $i<k<j$.

Proof $(\Rightarrow) C^{+}(Z)$ does not contain any of the points above a positive vertex $p_{k}^{+}$. Thus, if $p_{k}^{+}$is below $V_{i j}$ then $V_{i j}$ cannot be contained in $C^{+}(Z)$.

$(\Leftarrow) \quad$ Suppose that each $p_{k}^{+}$for $i<k<j$ lies on or above $V_{i j}$. Let $L$ be the piecewise linear curve going from $p_{i}$ to $p_{j}$, which goes through all positive vertices $p_{k}^{+}$between $p_{i}$ and $p_{j}$ and which bends only at these positive vertices. We see that $L$, and thus all points below $L$, is contained in $C^{+}(Z)$. Since each vertex on $L$ lies on or above $V_{i j}$, the entire curve $L$ lies on or above $V_{i j}$. So, $V_{i j}$ is contained in $C^{+}(Z)$ as claimed.

We define $C^{-}(Z)$ analogously to $C^{+}(Z)$ : Let $P^{-}$be the set of nonpositive vertices $p_{\ell}$. For any pair of consecutive vertices $p_{\ell_{i-1}}, p_{\ell_{i}}$ in $P^{-}$, let $C_{i}^{-}(Z)$ be the union of the convex hull of all vertices $p_{j}$ for $\ell_{i-1} \leq j \leq \ell_{i}$ and all points in $\mathbb{R}^{2}$ above this convex hull. Let $C^{-}(Z)=\bigcup C_{i}^{-}(Z)$. Then we have the following Lemma analogous to Lemma 1.6 above.

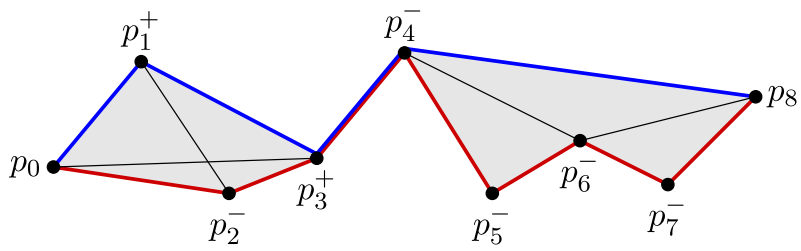

Fig. 3 The stability polygon $C(Z)=C^{+}(Z) \cap C^{-}(Z)$ consists of the two shaded regions and the chord $V_{34}=\overline{p_{3} p_{4}}$. The blue lines and points below the blue lines form $C^{+}(Z)$. The red lines and points above them form $C^{-}(Z)$. The 14 chords in $C(Z)$ represent the $14 Z$-stable modules. Also, $M_{47}$ is $Z$-semistable since $p_{6}^{-}$lies on the chord $V_{47}$ 
Lemma 2.7 For any $0 \leq i<j \leq n$, the chord $V_{i j}$ lies in $C^{-}(Z)$ if and only if $p_{\ell}^{-}$lies on or below $V_{i j}$ for all negative vertices $p_{\ell}^{-}$so that $i<\ell<j$.

Theorem 2.8 Given a central charge $Z: K A_{n}^{\varepsilon} \rightarrow \mathbb{C}$, the indecomposable $K A_{n}^{\varepsilon}$-module $M_{i j}$ is Z-semistable if and only if the chord $V_{i j}$ lies in the stability polygon $C(Z)=$ $C^{+}(Z) \cap C^{-}(Z) . M_{i j}$ is $Z$-stable if and only if it is $Z$-stable and $V_{i j}$ has no internal vertices.

Proof This follows immediately from Theorem 1.5 and Lemmas 1.6 and 1.7 above.

\subsection{Reineke's Conjecture}

We can now prove Reineke's conjecture for $A_{n}$ with any orientation.

Corollary 2.9 (Reineke's conjective for $A_{n}$ ) For a quiver $A_{n}^{\varepsilon}$ with any sign function $\varepsilon$, there is a standard linear stability condition making all indecomposable modules stable.

Proof By Theorem 1.8 above it suffices to find a standard central charge $Z$ (with $\mathbf{b}=$ $(1,1, \cdots, 1))$ so that the stability polygon $C(Z)$ is convex and so that no three vertices are collinear. Such a central charge is given by inscribing the stability polygon $C(Z)$ in the circle of radius $n / 2$ centered at $(n / 2,0)$ and letting the positive vertices $p_{k}^{+}$lie on the upper semi-circle and the negative $p_{\ell}^{-}$lie on the lower semi-circle. And $p_{0}, p_{n}$ on the $x$-axis.

More precisely: $p_{0}=(0,0), p_{n}=(n, 0)$,

$$
p_{s}^{\delta}=\left(s, \delta \sqrt{n^{2} / 4-(s-n / 2)^{2}}\right)
$$

for all $0<s<n$. See Fig. 4 .

Fig. 4 Proof of Reineke's conjecture: The stability polygon $C(Z)$ is convex with no three vertices collinear. So, all chords $V_{i j}$ are stable

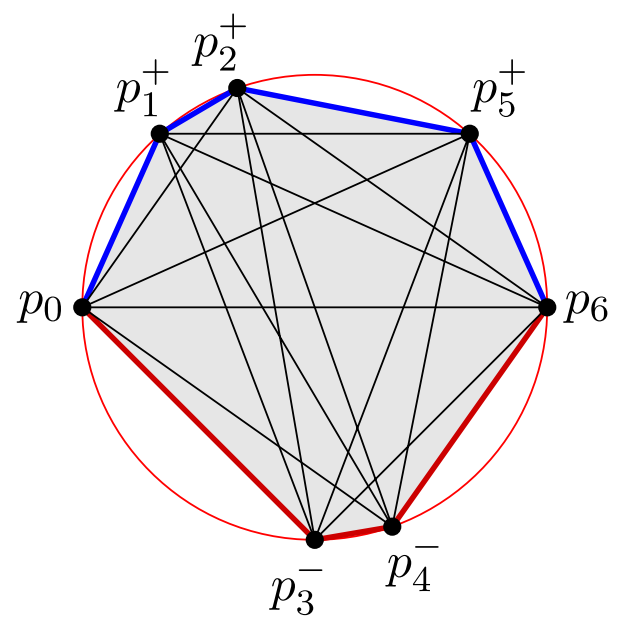




\section{Linear Wire Diagrams for $A_{n}$}

We briefly discuss the wire diagrams of type $A_{n}$. Given a central charge $Z(x)=\mathbf{a} \cdot x+i \mathbf{b} \cdot x$ for the quiver $A_{n}^{\varepsilon}$, the wires $L_{i} \subseteq \mathbb{R}^{2}, i=0, \cdots, n$ are defined to be the graphs of the functions $f_{i}: \mathbb{R} \rightarrow \mathbb{R}$ given by:

$$
f_{i}(t)=t\left(m-b_{1}-b_{2}-\cdots-b_{i}\right)+a_{1}+\cdots+a_{i}
$$

where $m$ is any convenient real number. The value of $m$ is not important since $f_{j}-f_{i}$ is independent of $m$. For every $0 \leq i<j \leq n$ let $t_{i j}$ be the $x$-coordinate of the intersection point $L_{i} \cap L_{j}$, i.e., the solution of the equation $f_{i}\left(t_{i j}\right)=f_{j}\left(t_{i j}\right)$. This is:

$$
t_{i j}=\frac{a_{i+1}+\cdots+a_{j}}{b_{i+1}+\cdots+b_{j}}=\sigma_{Z}\left(M_{i j}\right)=\text { slope of } V_{i j} .
$$

We have the following easy theorem.

Theorem 3.1 For $Z$ a central charge on $K A_{n}^{\varepsilon}, M_{i j}$ is $Z$-stable if and only if the following two conditions hold.

(1) For all $i<k<j$ with $\varepsilon_{k}=+, f_{k}\left(t_{i j}\right)>f_{i}\left(t_{i j}\right)=f_{j}\left(t_{i j}\right)$. Equivalently, $L_{k}$ lies above the point $L_{i} \cap L_{j}$.

(2) For all $i<\ell<j$ with $\varepsilon_{\ell}=-, f_{\ell}\left(t_{i j}\right)<f_{i}\left(t_{i j}\right)=f_{j}\left(t_{i j}\right)$, i.e., $L_{\ell}$ passes under $L_{i} \cap L_{j}$.

Proof This follows from Theorem 1.5 since (1) is equivalent to the condition that $p_{k}$ lies above the chord $V_{i j}$ and (2) is equivalent to the condition that $p_{\ell}$ lies below $V_{i j}$.

Proof of equivalence for (1): Since $f_{k}-f_{i}$ has negative slope and becomes 0 at $t_{i k}$, $f_{k}\left(t_{i j}\right)>f_{i}\left(t_{i j}\right)$ if and only if the slope of $V_{i k}$ which is $t_{i k}$ is greater than $t_{i j}$, the slope of $V_{i j}$. But this condition is equivalent to $p_{k}$ being above $V_{i j}$. The proof for (2) is similar.

Figure 5 shows the use of colors to determine stability. $M_{i j}$ is stable if $L_{i} \cap L_{j}$ is below all positive wires (in blue) of intermediate slope (between those of $L_{i}, L_{j}$ ) and above all negative wires (in red) of intermediate slope. For the wire diagram in Fig. 2 in the introduction we see that, in that example, all modules are stable except $M_{03}=I_{2}$.

\section{Wall Crossing and Maximal Green Sequences}

We recall the wall crossing version of stability from Bridgeland [3, 4], Derksen-Weyman $[11,18]$ which define a maximal green sequence. See $[6,16]$ for details of this particular

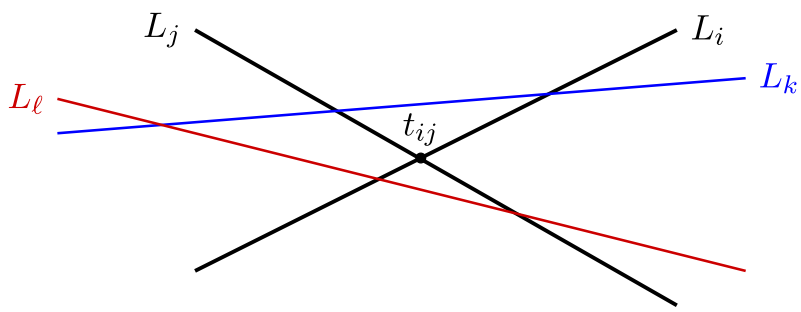

Fig. $5 M_{i j}$ is stable when all positive lines $L_{k}$ for $i<k<j$ (in blue) are above and all negative lines $L_{\ell}$ for $i<\ell<j$ (in red) are below $L_{i} \cap L_{j}$ 
formulation given by a green path passing through a finite sequence of "walls" $D(M)$. In this section, $\Lambda=K Q$ is the path algebra of an arbitrary acyclic quiver $Q$.

\subsection{Semistability Sets $D(M)$}

The relationship between linear stability conditions $Z$ and walls $D(M)$ defined below rests on the following observation.

Remark 4.1 A $\Lambda$-module $M$ is $Z$-stable for $Z(x)=\mathbf{a} \cdot x+i \mathbf{b} \cdot x$ with slope $\sigma_{Z}(M)=0$ if and only if $\mathbf{a} \cdot \underline{\operatorname{dim}} M=0$ and $\mathbf{a} \cdot \underline{\operatorname{dim}} M^{\prime}>0$ for all (nonzero) proper submodules $M^{\prime} \subset M$.

Definition 4.2 Let $M$ be a finitely generated module over $\Lambda=K Q$. The semistability set $D(M)$ of $M$ is defined to be the set

$$
D(M):=\left\{x \in \mathbb{R}^{n}: x \cdot \underline{\operatorname{dim}} M=0, x \cdot \underline{\operatorname{dim}} M^{\prime} \leq 0 \forall M^{\prime} \subseteq M\right\} .
$$

The stability set of $M$, denoted int $D(M)$, is defined to be the subset of $D(M)$ of points $x$ so that $x \cdot \operatorname{dim} M^{\prime}<0$ for all nonzero $M^{\prime} \subsetneq M$. Let $\partial D(M)=D(M)-\operatorname{int} D(M)$. We call $\partial D(M)$ the boundary of $D(M)$. The sets $D(M)$ are also called walls since they divide $\mathbb{R}^{n}$ into "chambers" as we explain below.

Note that $D(M)$ is a convex subset of the hyperplane

$$
H(M)=\underline{\operatorname{dim}} M^{\perp}=\left\{x \in \mathbb{R}^{n}: x \cdot \underline{\operatorname{dim}} M=0\right\} .
$$

For example, when $M=S_{i}$ is simple, we have $D\left(S_{i}\right)=H\left(S_{i}\right)=\left\{x \in \mathbb{R}^{n}: x_{i}=0\right\}$.

Remark 3.1 translates into the following condition on the linear path

$$
\lambda_{Z}(t):=\mathbf{b} t-\mathbf{a}
$$

corresponding to the central charge $Z(x)=\mathbf{a} \cdot x+i \mathbf{b} \cdot x$.

Proposition 4.3 The linear path $\lambda_{Z}$ crosses the wall $D(M)$ at time $t_{0}$ if and only if $M$ is $Z$-semistable with slope $t_{0}$. Furthermore $M$ is $Z$-stable if and only if $\lambda_{Z}\left(t_{0}\right) \in \operatorname{int} D(M)$.

Proof Since $\mathbf{b} \cdot \underline{\operatorname{dim}} M>0$, there is a unique $t_{0} \in \mathbb{R}$ so that $\lambda_{Z}\left(t_{0}\right) \in H(M)$ or, equivalently, $\lambda_{Z}\left(t_{0}\right) \cdot \operatorname{dim} M=0$. Solving for $t_{0}$ we get $t_{0}=\sigma_{Z}(M)$. If $M$ is $Z$-semistable then, for any $M^{\prime} \subseteq M$, we have $t_{1}=\sigma_{Z}\left(M^{\prime}\right) \geq \sigma_{Z}(M)=t_{0}$. Since $\mathbf{b} \cdot \underline{\operatorname{dim}} M^{\prime}>0$, we get:

$$
0=\lambda_{Z}\left(t_{1}\right) \cdot \underline{\operatorname{dim}} M^{\prime}=\left(t_{1}-t_{0}\right) \mathbf{b} \cdot \underline{\operatorname{dim}} M^{\prime}+\lambda_{Z}\left(t_{0}\right) \cdot \underline{\operatorname{dim}} M^{\prime} \geq \lambda_{Z}\left(t_{0}\right) \cdot \underline{\operatorname{dim}} M^{\prime} .
$$

Therefore $\lambda_{Z}\left(t_{0}\right) \in D(M)$. The same calculation proves the converse. This proves the first statement. The second statement follow from this and the definition of int $D(M)$.

Example 4.4 For example, in the standard case $\mathbf{b}=(1,1, \cdots, 1)$, the linear path $\gamma_{Z}$ crosses the simple walls $D\left(S_{i}\right)$ in increasing order of the coordinates $a_{i}$ since $\sigma_{Z}\left(S_{i}\right)=a_{i}$. For example, $\gamma_{1}(t)=(1,-1)+(t, t)$ in Fig. 6 below goes through $D\left(S_{1}\right)$, the $y$-axis, at time $t=a_{1}=-1$ then $D\left(S_{2}\right)$, the $x$-axis, at time $t=a_{2}=1$.

We need the following important observation from [16]. Recall that a module $M$ is called a brick if every nonzero endomorphism of $M$ is an automorphism.

Proposition 4.5 If the stability set int $D(M)$ is nonempty, then $M$ is a brick. 
Fig. 6 Semistability sets $D(M)$

for $K A_{2}^{-}$where $A_{2}^{-}: 1 \rightarrow 2$

with three green paths $\gamma_{1}, \gamma_{2}, \gamma_{3}$.

Since $S_{2} \subset P_{1}$,

$D\left(P_{1}\right)=\left\{x \in \mathbb{R}^{2} \mid x \cdot(1,1)=0, x \cdot(0,1) \leq 0\right\}$.

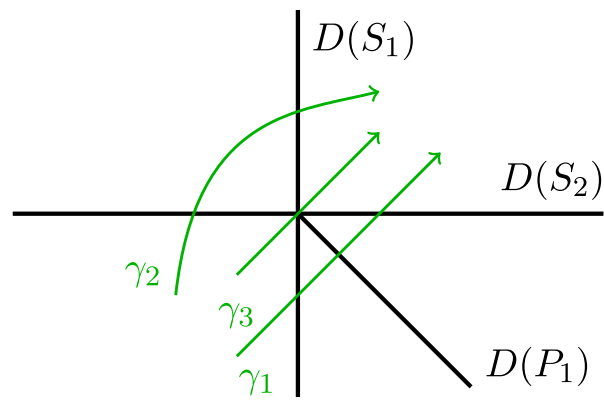

The simplest nontrivial example is the three dimensional algebra $\mathrm{KA}_{2}^{-}$with sign function $\varepsilon=(0,-, 0)$. This is the quiver $1 \rightarrow 2$. There are three indecomposable right $K A_{2}^{-}$modules $S_{1}, S_{2}, P_{1}$. The semistability sets $D\left(S_{1}\right), D\left(S_{2}\right), D\left(P_{1}\right) \subset \mathbb{R}^{2}$ are shown in Fig. 6 .

Definition 4.6 By a green path for $\Lambda$ we mean a smooth $\left(C^{1}\right)$ path $\gamma: \mathbb{R} \rightarrow \mathbb{R}^{n}$ having the following properties.

(1) $\gamma(t)$ has all coordinates negative for $t<<0$.

(2) $\gamma(t)$ has all coordinates positive for $t>>0$.

(3) Whenever $\gamma\left(t_{0}\right) \in D(M)$, the directional derivative of $\gamma$ in the direction $\operatorname{dim} M$ is positive, i.e.,

$$
\underline{\operatorname{dim}} M \cdot \frac{d \gamma}{d t}\left(t_{0}\right)>0 .
$$

We say that $\gamma$ passes through the wall $D(M)$ in the green direction if (3) holds.

Three examples of green paths are drawn in Fig. 6 with $\gamma_{1}, \gamma_{3}$ linear. The vector $(1,1)$ is always green. So, we see that each $\gamma_{i}$ passes through the walls in the green direction.

The path $\gamma(t)=(t, t, \cdots, t)\left(\gamma_{3}\right.$ in Fig. 6) is always green. More generally, any linear function $\lambda(t)=\mathbf{a}+\mathbf{b} t$ for $\mathbf{a}, \mathbf{b} \in \mathbb{R}^{n}$ is a green path if all coordinates of $b$ are positive. We call such a function a linear green path. We say that $\lambda=\mathbf{a}+\mathbf{b} t$ is a standard linear path if $\mathbf{b}=(1,1, \cdots, 1)$. (The corresponding central charge is $Z(x)=-\mathbf{a} \cdot x+i \mathbf{b} \cdot x$.)

Definition 4.7 Given a green path $\gamma$ for $\Lambda$, a $\Lambda$-module $M$ and $t_{0} \in \mathbb{R}$, the pair $\left(M, t_{0}\right)$ is called $\gamma$-stable, resp. $\gamma$-semistable, if $\gamma\left(t_{0}\right) \in \operatorname{int} D(M)$, resp $\gamma\left(t_{0}\right) \in D(M)$.

\subsection{Properties of Green Paths}

One of the fundamental properties which holds for any $\Lambda$ is the following.

Lemma 4.8 If $\gamma$ is a green path and $\left(M, t_{0}\right)$ is $\gamma$-stable, then $\left\{t_{0}\right\}=\gamma^{-1} H(M)$.

This implies that $t_{0}$ is uniquely determined. We denote it $t_{M}$. The proof of Lemma 3.8 follows the proof of Theorem 3.10 below.

Definition 4.9 A green path $\gamma$ will be called finite if there are only finitely many modules $M_{1}, \cdots, M_{m}$ (up to isomorphism) for which $\gamma$ passes through $D\left(M_{i}\right)$ and if, furthermore, $\gamma$ meets the interior of each $D\left(M_{i}\right)$ at distinct times $t_{i}$ and $t_{1}<t_{2}<\cdots<t_{m}$. By a maximal green sequence (MGS) for $\Lambda$ we mean a finite ordered sequence $M_{1}, \cdots, M_{m}$ 
given by some finite green path $\gamma$. The MGS will be called linear, resp. standard linear, if it is given by a linear green path, resp. standard linear path.

For any green path we have: $\operatorname{Hom}_{\Lambda}\left(M_{i}, M_{j}\right)=0$ whenever $i<j$. This is Corollary 3.11 which follows easily from the following theorem that we will need later in this paper. (See $[6,16,17]$ for more details and other equivalent definitions of a maximal green sequence.)

Theorem 4.10 Suppose that $\gamma$ is a green path for $\Lambda$ and $\left(M, t_{0}\right)$ is $\gamma$-stable. Then $\gamma$ crosses the hyperplanes of proper submodules $M^{\prime} \subsetneq M$ after $t_{0}$ and it crosses the hyperplanes of proper quotient modules $M^{\prime \prime}$ before $t_{0}$. More precisely:

(a) Whenever $\gamma\left(t^{\prime}\right) \in H\left(M^{\prime}\right)$ for some $M^{\prime} \subsetneq M$ then $t_{0}<t^{\prime}$.

(b) Whenever $\gamma\left(t^{\prime \prime}\right) \in H\left(M^{\prime \prime}\right)$ for some proper quotient module $M^{\prime \prime}$ of $M$ then $t^{\prime \prime}<t_{0}$.

Proof (a) For every $x$ in the hyperplane $H(M)=(\underline{\operatorname{dim}} M)^{\perp}$, let $h_{x}: \mathbb{R} \rightarrow \mathbb{R}^{n}$ be the linear function $h_{x}(t)=x+t \underline{\operatorname{dim}} M$. The image of $h_{x}$ is a straight line perpendicular to $H(M)$ passing through $x=h_{x}(0)$. Let $t=\mu(x) \in \mathbb{R}$ be the smallest real number so that $h_{x}(t) \in H\left(M^{\prime}\right)$ for some proper submodule $M^{\prime}$ of $M$. In other words,

$$
x \cdot \underline{\operatorname{dim}} M^{\prime}+t \underline{\operatorname{dim}} M \cdot \underline{\operatorname{dim}} M^{\prime}=0 .
$$

Since $\underline{\operatorname{dim}} M \cdot \underline{\operatorname{dim}} M^{\prime} \geq \operatorname{dim} M^{\prime}>0$, we can divide to get:

$$
\mu(x)=\min _{M^{\prime}} \frac{-x \cdot \underline{\operatorname{dim}} M^{\prime}}{\underline{\operatorname{dim}} M \cdot \underline{\operatorname{dim}} M^{\prime}}
$$

Since there are only finitely many dimension vectors of the form $\underline{\operatorname{dim}} M^{\prime}, \mu$ is the minimum of a finite collection of linear functions. So, $\mu$ is continuous.

For every $x \in H(M)$ we have the following.

(1) $h_{x}(\mu(x)) \in H\left(M^{\prime}\right)$ for some $M^{\prime} \subsetneq M$.

(2) $h_{x}(t) \cdot \underline{\operatorname{dim}} M^{\prime} \leq 0$ for all $t \leq \mu(x)$ and all $M^{\prime} \subsetneq M$.

For pairs $x, M^{\prime}$ which occur in (1) it follows that:

(3) $h_{x}(\mu(x)) \in D\left(M^{\prime}\right)$

(4) $h_{x}(t) \cdot \underline{\operatorname{dim}} M^{\prime}>0$ for all $t>\mu(x)$.

Since $x=h_{x}(0)$ we see that

(5) $x$ is in the interior of $D(M)$ if and only if $\mu(x)>0$.

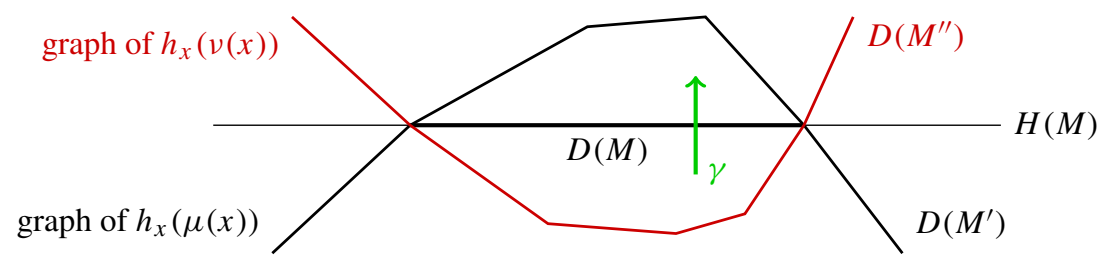

If $\gamma$ is a green path, it must cross the graph of the function $x \mapsto h_{x}(\mu(x))$ at some point. By (3), this graph is a union of semistability sets $D\left(M^{\prime}\right)$. So, the "green" condition (4.6(3)) means it passes from below to above. Thus, $\gamma$ can only cross this graph once from below to 
above. By definition of $\mu$, every hyperplane $H\left(M^{\prime}\right)$ for every $M^{\prime} \subsetneq M$ lies on or above this graph. So, if $\gamma\left(t^{\prime}\right) \in H\left(M^{\prime}\right)$ for any such $M^{\prime}$, it must be after $\gamma$ crosses this graph.

Given that $\left(M, t_{0}\right)$ is $\gamma$-stable, $\gamma\left(t_{0}\right)$ must be in the interior of $D(M)$ which, by (5), is below the graph of the function $h_{x}(\mu(x))$. So, $\gamma$ will hit the graph of the function after time $t_{0}$. This implies $t^{\prime}>t_{0}$. So, (a) holds.

The proof of (b) is similar using $v(x)$, the largest real number so that $h_{x}(v(x)) \in H\left(M^{\prime \prime}\right)$ for some proper quotient $M^{\prime \prime}$ of $M$.

Proof of Lemma 3.8 The statement is that, for $\left(M, t_{0}\right) \gamma$-stable, $t_{0}$ is unique. When $\gamma$ crosses $D(M)$, it must already have crossed the graph of $h_{x}(v(x))$ and has not yet crossed the graph of $h_{x}(\mu(x))$. So, it can never cross $H(M)-D(M)$ which is below the first graph and above the second. Also, $\gamma$ can cross $D(M)$ only once in the green direction.

Corollary 4.11 Let $\left(M_{1}, t_{1}\right),\left(M_{2}, t_{2}\right)$ be stable pairs for a green path $\gamma$ with $t_{1}<t_{2}$. Then $\operatorname{Hom}_{\Lambda}\left(M_{1}, M_{2}\right)=0$.

Proof Suppose there is a nonzero morphism $f: M_{1} \rightarrow M_{2}$. Let $X=f\left(M_{1}\right) \subseteq M_{2}$. The path $\gamma$ must pass through the hyperplane $H(X)$ at least once since it starts from its negative side and ends on its positive side. Let $t_{x}$ be one of these times. Then, by (b) in Theorem 4.10, we have $t_{x}<t_{1}$ if $X$ is a proper quotient of $M_{1}$ and $t_{x}=t_{1}$ by Lemma 4.8 if $X=M_{1}$. Similarly, by (a) and Lemma $4.8, t_{2} \leq t_{x}$ since $X \subseteq M_{2}$. This contradicts the assumption that $t_{1}<t_{2}$.

Remark 4.12 It is shown in [16] for $\Lambda$ hereditary and in [17] for $\Lambda$ cluster-tilted of finite type (which includes all examples in this paper) that maximal green sequences are characterized by this hom-orthogonality condition. More precisely, a sequence of bricks $M_{1}, \cdots, M_{m}$ is a MGS if and only if $\operatorname{Hom}_{\Lambda}\left(M_{i}, M_{j}\right)=0$ for $i<j$ and the sequence $\left(M_{i}\right)$ is maximal with this property.

Lemma 4.8 and Theorem 4.10 give necessary conditions for $\left(M, t_{0}\right)$ to be $\gamma$-stable. However, we need necessary and sufficient conditions such as the following.

Theorem 4.13 Let $\gamma$ be a green path and $\lambda(t)=\boldsymbol{a}+\boldsymbol{b} t$ any linear green path for $\Lambda$ so that

$$
\lambda\left(t_{0}\right)=\gamma\left(t_{0}\right) \in H(M)
$$

for some $\Lambda$-module $M$ and $t_{0} \in \mathbb{R}$. Then $\left(M, t_{0}\right)$ is $\gamma$-stable if and only if, for every nonzero proper submodule $M^{\prime} \subsetneq M$, there is a $t^{\prime}>t_{0}$ so that $\lambda\left(t^{\prime}\right) \in H\left(M^{\prime}\right)$.

$$
\left(\forall M^{\prime} \subsetneq M\right)\left(\exists t^{\prime}>t_{0}\right) \lambda\left(t^{\prime}\right) \in H\left(M^{\prime}\right)
$$

Proof Let $Z$ be the central charge given by $Z(x)=-\mathbf{a} \cdot x+i \mathbf{b} \cdot x$. Then the condition $\lambda\left(t_{0}\right) \in H(M)$ is equivalent to the equation $t_{0}=\sigma_{Z}(M)$ and Eq. 4.1 above is equivalent to $t^{\prime}=\sigma_{Z}\left(M^{\prime}\right)>t_{0}$. This equation for all nonzero $M^{\prime} \subsetneq M$ is equivalent to the statement that $\lambda\left(t_{0}\right)=\gamma\left(t_{0}\right) \in \operatorname{int} D(M)$. See Proposition 4.3 and its proof for details.

Remark 4.14 Equivalently, $\left(M, t_{0}\right)$ is $\gamma$-stable if and only if, for every nonzero proper quotient module $M^{\prime \prime}$ of $M$, there is a $t^{\prime \prime}<t_{0}$ so that $\lambda\left(t^{\prime \prime}\right) \in H\left(M^{\prime \prime}\right)$. 


\subsection{Affine Quivers of Type $\tilde{A}$}

In this paper we are interested in quivers of type $\widetilde{A}_{a, b}$. These have $n=a+b$ vertices labeled with integers modulo $n$ and $n$ arrows arranged in a circle with $a$ arrows going clockwise and $b$ going counterclockwise. There are $2^{n}$ possible orientations of such a quiver which we indicate with a sign function $\varepsilon:[1, n] \rightarrow\{+,-\}$ or, equivalently, an $n$-periodic sign function $\varepsilon: \mathbb{Z} \rightarrow\{+,-\}$. The sign $\varepsilon_{i}$ is positive or negative depending on whether there is an arrow $i \leftarrow i+1$ or $i \rightarrow i+1$, respectively.

For example we have:

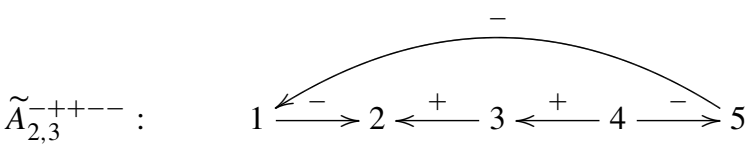

The exponent indicates the sign function.

All quivers of type $\widetilde{A}_{a, b}, a, b \geq 1$, are of infinite type, that is, they have infinitely many indecomposable representations up to isomorphism. (See [12] for a description of all indecomposable representations of type $\widetilde{A}_{a, b}$.) However, in a maximal green sequence it is well-known [10] that only the rigid modules occur. These are the modules $M_{i j}, i<j$ which we now describe.

We write the finite cyclic quiver as an infinite $n$-periodic linear quiver:

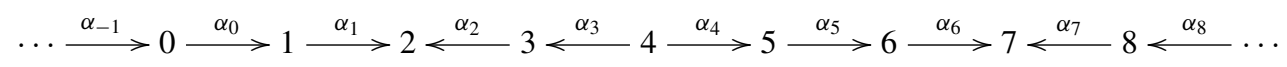

with vertices and arrows labeled with integers so that the direction of $\alpha_{i}$ is the same as that of $\alpha_{i+k n}$ for any integer $k$. The direction of these arrows is given by the $n$-periodic version of the sign function

$$
\varepsilon=(\cdots,-,-,-,+,+,-,-,-,+,+, \cdots)
$$

which we denote $\varepsilon=\left(\varepsilon_{1}, \cdots, \varepsilon_{n}\right)$ which is $(-,+,+,-,-)$ in this case. This periodic linear quiver is the universal covering of the quiver of type $\widetilde{A}_{2,3}^{-++--}$from Eq. 4.2. The representation $M_{i j}$ of $\widetilde{A}_{a, b}^{\varepsilon}$ is "pushed-down" from the indecomposable representation $\widetilde{M}_{i j}$ of the infinite quiver with dimension vector $e_{i+1}+\cdots+e_{j}$, just as in the finite case. The push-down $\bar{M}$ of a representation $M$ of the infinite quiver is given by $\bar{M}_{i}=\bigoplus_{k} M_{i+k n}$ with negative arrows $i \rightarrow i+1$ inducing $\sum_{k} M_{\alpha_{i+k n}}: M_{i+k n} \rightarrow M_{i+k n+1}$ and similarly for positive arrows.

\section{Wire Diagrams for $\tilde{A}_{a, b}$}

Any green path can be written as

$$
\gamma(t)=t \mathbf{b}(t)-\mathbf{a}(t)
$$

where $\mathbf{a}, \mathbf{b}: \mathbb{R} \rightarrow \mathbb{R}^{n}$ are $C^{1}$ functions with velocity vectors $\mathbf{a}^{\prime}(t), \mathbf{b}^{\prime}(t)$ equal to zero for $|t|$ large (giving four vectors $\mathbf{a}(\infty), \mathbf{a}(-\infty), \mathbf{b}(\infty), \mathbf{b}(-\infty)$ ) and so that $b_{i}(t)>0$ for all $t$ and $i$. For example, we could let $\mathbf{b}(t)$ be the constant vector $\mathbf{b}=(1,1, \cdots, 1)$ and let $\mathbf{a}(t)=t \mathbf{b}-\gamma(t)$. Although the decomposition of $\gamma$ into the two parts $t \mathbf{b}(t)$ and $-\mathbf{a}(t)$ is 
not unique, it gives a very useful interpretation of $\gamma$ for quivers of type $A_{n}$ and $\widetilde{A}_{a, b}$. Also, in Theorem 4.13 we can take the linear green path $\lambda$ to be $\lambda_{t_{0}}$ given by

$$
\lambda_{t_{0}}(t):=t \mathbf{b}\left(t_{0}\right)-\mathbf{a}\left(t_{0}\right)
$$

since, clearly, $\lambda_{t_{0}}\left(t_{0}\right)=\gamma\left(t_{0}\right)$.

\subsection{Slope}

Given a fixed decomposition $\gamma(t)=t \mathbf{b}(t)-\mathbf{a}(t)$, we can define the slope of any nonzero $\Lambda$-module $M$ at time $t$ by

$$
\sigma_{t}(M):=\frac{\mathbf{a}(t) \cdot \underline{\operatorname{dim}} M}{\mathbf{b}(t) \cdot \underline{\operatorname{dim}} M} .
$$

We have the following easy calculation.

$$
\sigma_{t}(M)=t \Leftrightarrow \gamma(t) \cdot \underline{\operatorname{dim}} M=0 \Leftrightarrow \gamma(t) \in H(M) .
$$

The following characterization of stable pairs follows from Theorem 4.13 using $\lambda=\lambda_{t_{0}}$.

Theorem 5.1 Let $\gamma$ be a green path with slope function $\sigma_{t}$. Then $\left(M, t_{0}\right)$ is a $\gamma$-stable pair, i.e., $\gamma\left(t_{0}\right) \in \operatorname{int} D(M)$, if and only if the following conditions are satisfied.

(1) $t_{0}=\sigma_{t_{0}}(M)$

(2) $\sigma_{t_{0}}\left(M^{\prime}\right)>t_{0}$, for all nonzero $M^{\prime} \subsetneq M$.

Note that, since the numerator of $\sigma_{t_{0}}(M)-\sigma_{t_{0}}\left(M^{\prime}\right)$ is equal to the numerator of $\sigma_{t_{0}}\left(M / M^{\prime}\right)-\sigma_{t_{0}}(M)$, condition (2) is equivalent to the following condition:

(3) $\sigma_{t_{0}}\left(M^{\prime \prime}\right)<t_{0}$ for all proper quotient modules $M^{\prime \prime}$ of $M$.

Proof By Eq. 5.2, (1) is equivalent to the condition $\gamma\left(t_{0}\right) \in H(M)$. Now apply Theorem 3.13 using $\lambda=\lambda_{t_{0}}$ defined in Eq. 5.1. For any $M^{\prime} \subsetneq M$, the formula for $\sigma_{t_{0}}$ shows that $t^{\prime}=\sigma_{t_{0}}\left(M^{\prime}\right)$ is the unique real number so that $\lambda_{t_{0}}\left(t^{\prime}\right) \in H\left(M^{\prime}\right)$. Therefore, (2) is equivalent to the condition $t^{\prime}>t_{0}$ in Theorem 4.13 which is equivalent to $\left(M, t_{0}\right)$ being $\gamma$-stable.

Next, we use the well-known fact that, for $\Lambda$ hereditary, the modules $M$ in any maximal green sequence are exceptional which means that ${\underset{\sim}{\tilde{A}}}_{a, b}(M)=K$ and $\operatorname{Ext}_{\Lambda}^{1}(M, M)=0$. We also use the well-known fact that $\Lambda$ of type $\widetilde{A}_{a, b}$ is a "gentle algebra" in which all exceptional modules are "string modules" of the form $M_{i j}$ for $i<j$ with $j-i$ not divisible by $n$ with dimension vector

$$
\underline{\operatorname{dim}} M_{i j}=\beta_{i j}=\sum_{k=i+1}^{j} e_{k}
$$

where $e_{k}$ is the $p$-th unit vector for $p \in[1, n]$ congruent to $k$ modulo $n$. The indices $i, j$ satisfy one more condition: when $\varepsilon_{i}=\varepsilon_{j}$, we must have $|i-j|<n$, otherwise $M_{i j}$ is not exceptional. This condition also appears later in Proposition 6.7.

\subsection{The Functions $f_{i}(t)$}

For $\Lambda$ of type $\widetilde{A}_{a, b}$, the "wires" in our "wire diagram" are the graphs of functions $f_{i}: \mathbb{R} \rightarrow$ $\mathbb{R}, i \in \mathbb{Z}$, given by

$$
f_{i}(t)=m_{i}(t) t+c_{i}(t)
$$


where $c_{i}=a_{1}+a_{2}+\cdots+a_{i}$, taking indices modulo $n$, and $m_{i}=m_{0}-b_{1}-b_{2}-\cdots-b_{i}$ for some fixed smooth function $m_{0}: \mathbb{R} \rightarrow \mathbb{R}$.

Lemma 5.2 For $i<j$ and $t_{0} \in \mathbb{R}$, the following are equivalent.

(1) $f_{i}\left(t_{0}\right)=f_{j}\left(t_{0}\right)$.

(2) $\gamma\left(t_{0}\right) \in H\left(M_{i j}\right)$.

Proof Since $f_{i}(t)-f_{j}(t)=\left(b_{i+1}+\cdots+b_{j}\right) t-\left(a_{i+1}+\cdots+a_{j}\right)$ the values of $t_{0}$ so that $f_{i}\left(t_{0}\right)=f_{j}\left(t_{0}\right)$ are given by

$$
t_{0}=\frac{a_{i+1}+\cdots+a_{j}}{b_{i+1}+\cdots+b_{j}}=\frac{\mathbf{a}(t) \cdot \underline{\operatorname{dim}} M_{i j}}{\mathbf{b}(t) \cdot \underline{\operatorname{dim}} M_{i j}}=\sigma_{t_{0}}\left(M_{i j}\right)
$$

So, (1) is equivalent to $t_{0}=\sigma_{t_{0}}\left(M_{i j}\right)$ which, by Eq. 5.2, is equivalent to (2).

Let $T_{i j}=\left\{t_{i j}\right\}$ denote the set of all real numbers so that $f_{i}\left(t_{i j}\right)=f_{j}\left(t_{i j}\right)$. Thus $T_{i j}=$ $T_{j i}=\gamma^{-1} H\left(M_{i j}\right)$ by Lemma 5.2 above. Since $f_{i}(t)<f_{j}(t)$ for $t<<0$ and $f_{i}(t)>f_{j}(t)$ for $t>>0$ (when $i<j$ ), $T_{i j}$ is always nonempty. The smallest and largest elements of $T_{i j}$ will be denoted $t_{i j}^{0}$ and $t_{i j}^{1}$ respectively.

The order theorems, from Section 4.2, which hold for any finite dimensional algebra imply the following for $\widetilde{A}_{a, b}$.

Lemma 5.3 Let $M_{i j}$ be $\gamma$-stable for a green path $\gamma$. Then $t_{i j} \in T_{i j}$ is unique.

Proof By Lemma 4.8, $\gamma^{-1} H\left(M_{i j}\right)=T_{i j}$ has only one element.

Theorem 5.4 Suppose that $t_{i j}$ is unique. Then $\left(M_{i j}, t_{i j}\right)$ is $\gamma$-stable if and only if

(1) for all $i<k<j$ with $\varepsilon_{k}=+$ we have $f_{k}\left(t_{i j}\right)>f_{i}\left(t_{i j}\right)$ and

(2) for all $i<\ell<j$ with $\varepsilon_{\ell}=-$ then $f_{\ell}\left(t_{i j}\right)<f_{i}\left(t_{i j}\right)$.

Remark 5.5 Paraphrased: $M_{i j}$ is stable if and only if $L_{i}, L_{j}$ meet at only one point $t_{i j}$ and all positive (blue) curves $L_{k}$ with $i<k<j$ go over that point and all negative (red) curves $L_{\ell}$ with $i<\ell<j$ go under that point.

Proof We again use Theorem 4.13 with $\lambda(t)=t \mathbf{b}\left(t_{i j}\right)-\mathbf{a}\left(t_{i j}\right)$. Let $L_{i}^{\lambda}, L_{j}^{\lambda}$, etc. denote the linear wires for $\lambda$. Let $t_{i j}^{\lambda}$ be the $x$-coordinate of $L_{i}^{\lambda} \cap L_{j}^{\lambda}$. If $L_{k}^{\lambda}$ lies above $L_{i}^{\lambda} \cap L_{j}^{\lambda}$ and $L_{\ell}^{\lambda}$ lies below $L_{i}^{\lambda} \cap L_{j}^{\lambda}$ then the $t$ coordinates of their intersection points are arranged:

$$
t_{i j}=t_{i j}^{\lambda}<t_{i k}^{\lambda}, t_{\ell j}^{\lambda}
$$

Also, $t_{i j}^{\lambda}<t_{\ell k}^{\lambda}$ when $\ell<k$. Since $\varepsilon_{k}=+$ and $\varepsilon_{\ell}=-, M_{i k} \subsetneq M_{i j}, M_{\ell j} \subsetneq M_{i j}$ and $M_{\ell k} \subsetneq M_{i j}$ when $\ell<k$. These are all of the proper indecomposable submodules of $M_{i j}$. So, by Theorem $4.13, M_{i j}$ is $\gamma$-stable.

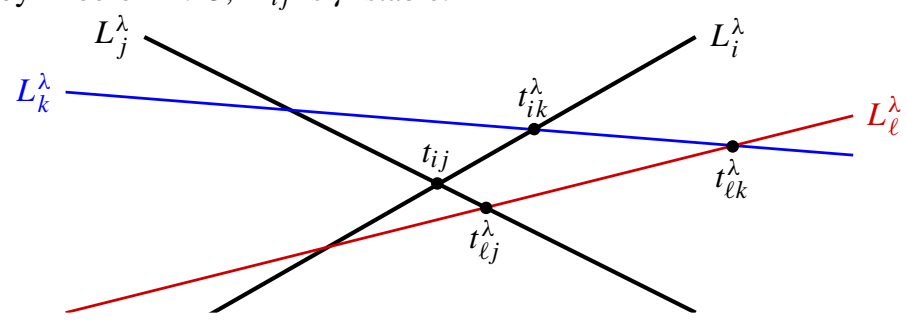


Conversely, if $M_{i j}$ is $\gamma$-stable then, by Theorem 4.13, $t_{i j}$ must be less than $t_{i k}^{\lambda}$ and $t_{\ell j}^{\lambda}$ for $i<k, \ell<j, \varepsilon_{k}=+, \varepsilon_{\ell}=-$. This implies that the straight line $L_{k}^{\lambda}$ goes above $L_{i}^{\lambda} \cap L_{j}^{\lambda}$ and $L_{\ell}^{\lambda}$ goes below $L_{i}^{\lambda} \cap L_{j}^{\lambda}$. But $\gamma\left(t_{i j}\right)=\lambda\left(t_{i j}\right)$. So, $f_{\ell}\left(t_{i j}\right)<f_{i}\left(t_{i j}\right)<f_{k}\left(t_{i j}\right)$ as claimed.

When $\gamma$ is linear, we can reinterpret Theorem 5.4 in terms of chords $V_{i j}$ as follows.

Corollary 5.6 Let $\gamma(t)=\boldsymbol{b} t-\boldsymbol{a}$ be the linear green path with $\boldsymbol{a}, \boldsymbol{b} \in \mathbb{R}^{n}$ constant with $b_{i}>0$ for every $i$, i.e., $\gamma=\lambda_{Z}$ for the central charge $Z$ given by $Z(x)=\boldsymbol{a} \cdot x+i \boldsymbol{b} \cdot x$. Let $p_{i} \in \mathbb{R}^{2}$ be given by

$$
p_{i}=\left(b_{1}+b_{2}+\cdots+b_{i}, a_{1}+a_{2}+\cdots+a_{i}\right)
$$

Let $V_{i j}$ be the line segment from $p_{i}$ to $p_{j}$. Then $M_{i j}$ is $\gamma$-stable if and only if the following two conditions are satisfied:

(1) For every $i<k<j$ with $\varepsilon_{k}=+$ the point $p_{k}$ lies above the line segment $V_{i j}$.

(2) For every $i<\ell<j$ with $\varepsilon_{\ell}=-$ the point $p_{\ell}$ lies below $V_{i j}$.

Proof The slope of $V_{i j}$, which is equal to $\sigma_{Z}\left(M_{i j}\right)=t_{i j}$, is less than $t_{i k}$ and $t_{\ell j}$, the slopes of $V_{i k}$ and $V_{\ell j}$, if and only if Conditions (1) and (2) hold.

Theorem 5.7 Let $M_{i j}$ be $\gamma$-stable for a green path $\gamma$. Then $t_{i j}$ is unique and we have the following.

(1) Suppose $i<k<j$ and $\varepsilon_{k}=+$. Then

$$
t_{k j}^{1}<t_{i j}<t_{i k}^{0} .
$$

(2) Similarly, for $i<\ell<j$, and $\varepsilon_{\ell}=-$ we have

$$
t_{i \ell}^{1}<t_{i j}<t_{\ell j}^{0} \text {. }
$$

Conversely, when (1), (2) both hold and $t_{i j}$ is unique we have:

$$
f_{\ell}\left(t_{i j}\right)<f_{i}\left(t_{i j}\right)=f_{j}\left(t_{i j}\right)<f_{k}\left(t_{i j}\right) .
$$

These three statements are illustrated in Fig. 7.

Proof As in the case of finite $A_{n}$ quivers, the positivity of $k$ implies that the $k$ th arrow points to the left: $M_{i k} \leftarrow \cdots$. Therefore, $M_{i k} \subsetneq M_{i j}$. By Theorem 4.10, $t_{i j}<t^{\prime}$ for any $t^{\prime} \in \gamma^{-1} H\left(M_{i k}\right)$. We are using the notation $t^{\prime}=t_{i k}$. So, $t_{i j}<t_{i k}$. The other three cases are
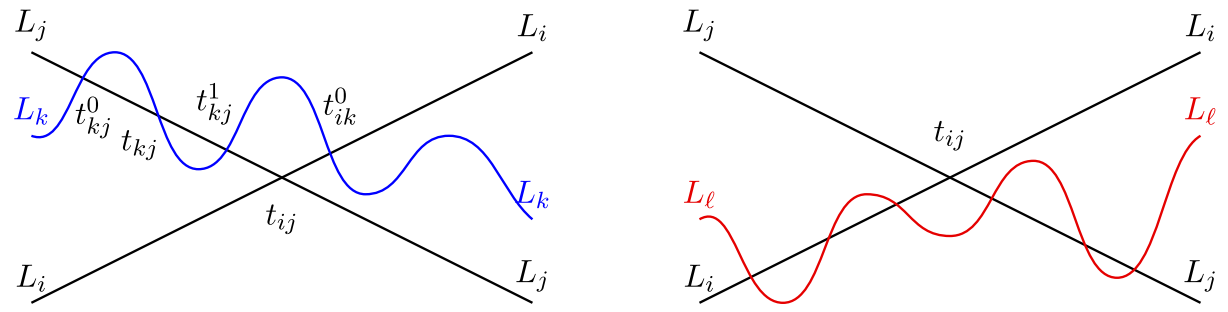

Fig. 7 The wires $L_{i}, L_{j}$ cross only once, at $t_{i j}$, since $M_{i j}$ is stable. The statement $t_{i j}<t_{i k}^{0}$ (the first crossing of $L_{i}, L_{k}$ ) is equivalent to saying that $L_{k}$ does not cross $L_{i}$ to the left of $t_{i j}$. Similarly $t_{k j}^{1}<t_{i j}$ says $L_{k}$ does not cross $L_{j}$ to the right of $t_{i j}$. (All three values of $t_{k j}$ are to the left of $t_{i j}$ ) 
similar. The converse statement follows by examination of Fig. 7. E.g., $L_{k}$ cannot go under $L_{i} \cap L_{j}$ without crossing $L_{i}$ to the left of $t_{i j}$.

Definition 5.8 If $i<k<j$ with $\varepsilon_{k}=+$ and either $t_{i k}^{0}<t_{i j}$ or $t_{i j}<t_{k j}^{1}$ we say that $k$ is a witness to the instability of $M_{i j}$. Similarly, $\ell$ is a witness to the instability of $M_{i j}$ if $i<\ell<j$ with $\varepsilon_{\ell}=-$ satisfies either $t_{\ell j}^{0}<t_{i j}$ or $t_{i j}<t_{i \ell}^{1}$.

Theorem 5.9 Let $M_{i j}$ be $\gamma$-stable for a green path $\gamma$. Then $t_{i j}$ is unique and we have the following.

(3) Whenever $i<k<\ell<j$ with $\varepsilon_{k}=+$, $\varepsilon_{\ell}=-$ we have:

$$
t_{k \ell}^{1}<t_{i j}
$$

(4) Whenever $i<\ell<k<j$ with $\varepsilon_{k}=+$, $\varepsilon_{\ell}=-$ we have:

$$
t_{i j}<t_{\ell k}^{0}
$$

Proof In Case (4), when $\ell<k$ and $\varepsilon_{k}=+, \varepsilon_{\ell}=-$, the arrow pattern is: $\rightarrow M_{\ell k} \leftarrow$ making $M_{\ell k}$ a proper submodule of $M_{i j}$. So, $t^{\prime}>t_{i j}$ for all $t^{\prime}=t_{\ell k} \in T_{\ell k}$ by Theorem 3.10 (Fig. 8). In Case (3), $M_{k \ell}$ is a proper quotient module of $M_{i j}$. So, $t^{\prime \prime}<t_{i j}$ for all $t^{\prime \prime}=t_{k \ell} \in T_{k \ell}$.

\subsection{Example: A Nonlinear Maximal Green Sequence}

The wire diagram in Fig. 9 shows an example of a nonlinear maximal green sequence for $A_{5}^{-+-+}$.

(1) This is a wire diagram since each curve is the graph of a continuous function $\mathbb{R} \rightarrow \mathbb{R}$, all crossing are transverse and the wires $L_{0}, \cdots, L_{5}$ are in ascending order on the left and descending order on the right.

(2) The diagram gives a maximal green sequence since all stable crossings are green (with lower numbered wire having higher slope).

(3) All modules $M_{i j}$ are stable since $L_{i}, L_{j}$ cross only once and the intermediate blue, resp. red, wires pass above, resp. below, the crossing point. E.g., $M_{05}$ is stable since $L_{2}, L_{4}$ go over the crossing point $L_{0} \cap L_{5}$ and $L_{1}, L_{3}$ go under that point.

(4) The diagram is nonlinear since, if it were linear, the wires $L_{0}, L_{5}$ could be moved to the left to make the crossings $t_{01}, t_{45}$, resp. $t_{02}, t_{35}$, line up with $t_{23}$, resp. $t_{14}$. But this would move the crossing $t_{05}$ to the left contradicting Pappus' Theorem.

Fig. 8 Case (4): if $M_{i j}$ is stable and $i<\ell<k<j$ with $\varepsilon_{k}=+, \varepsilon_{\ell}=-$, the graphs of $f_{k}$ $f_{\ell}$ do not cross on the left side of $t_{i j}$. (In this example, all three values of $t_{\ell k}$ are $>t_{i j}$ )

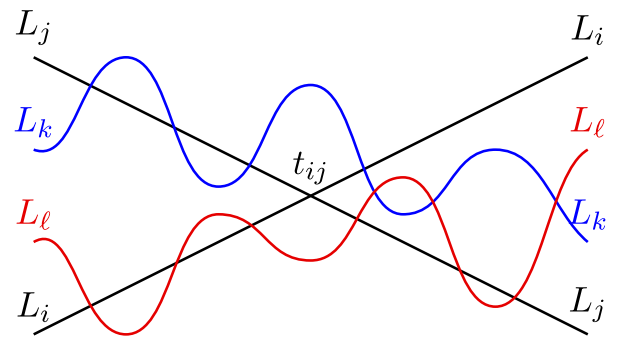




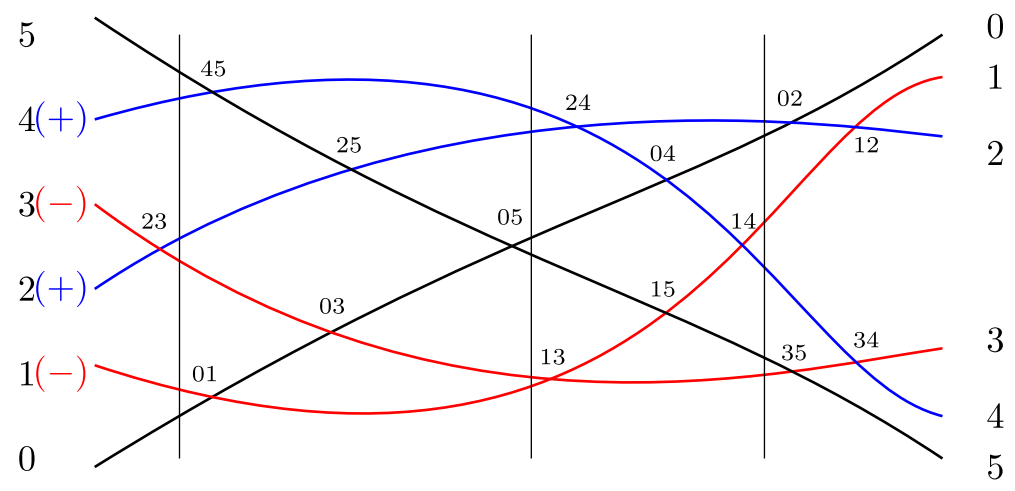

Fig. 9 Example of a nonlinear MGS on $A_{5}^{-+-+}: 1 \rightarrow 2 \leftarrow 3 \rightarrow 4 \leftarrow 5$. Theorem 5.4 shows that all modules $M_{i j}$ are stable. Pappus' Theorem implies that this arrangement of lines is nonlinear

This proves the following.

Theorem 5.10 For the quiver $A_{5}^{-+-+}$, Fig. 9 gives an example of a maximal green sequence of maximal length, 15, which is not given by any linear stability condition.

\section{Critical Line and Upper Bound}

In this section we introduce the critical line and use the order of the points on the critical line to determine which wire crossings can be stable. We then use this to find the upper bound on the length of a maximal green sequence by assuming that all of these crossings are indeed stable. In Section 7 we will show that this upper bound is actually attained.

Throughout this section we assume that $\gamma$ is a generic green path for a quiver of type $\widetilde{A}_{a, b}^{\varepsilon}$ with associated functions $f_{i}$ and wires $L_{i}$ which are the graphs of these functions. Note that, since $\gamma$ is generic, no three wires meet at any point. So, $M_{i j}$ is $(\gamma)$-stable if and only if it is $(\gamma)$-semistable. We will also say that the intersection $t_{i j}$ is stable in that case.

\subsection{The Critical Line}

Due to the periodicity of the wire diagram $\left(f_{i+n}(t)-f_{i}(t)=f_{j+n}(t)-f_{j}(t)\right.$ for all $\left.i, j\right)$, we can observe that $t_{0, n}^{0}=t_{1, n+1}^{0}=t_{2, n+2}^{0}=\ldots$, or more generally that $t_{i, i+n}^{0}=t_{j, j+n}^{0}$ for all $i, j$. This leads to the following definition.

Definition 6.1 Let $c^{0}=t_{0, n}^{0}$. The critical line is the line $t=c^{0}$, which is the left-most vertical line upon which all the intersections $L_{i} \cap L_{i+n}$ fall. We say $i \triangleleft j$ when $f_{i}\left(c^{0}\right)<$ $f_{j}\left(c^{0}\right)$ and $i \unlhd j$ when $f_{i}\left(c^{0}\right) \leq f_{j}\left(c^{0}\right)$.

The critical line is so called because we can use it to determine several conditions for stability of intersections, to be described shortly. Note that the signs of the lines in the wire diagram determine a sequence on the critical line, such as the one in Fig. 10. 


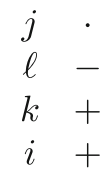

Fig. 10 Four points on the critical line with $\varepsilon_{i}=\varepsilon_{k}=+$ and $\varepsilon_{\ell}=-$. The figure indicates $i \triangleleft k \triangleleft \ell \triangleleft j$ but does not preclude the presence of other points on the critical line above, below or between these points

\subsection{Lemmas}

For each $i, j$, we will determine all $p$ so that $M_{i, j+p n}$ can be stable. More precisely we show that $M_{i, j+p n}$ is unstable (not semistable) for all but two values of $p$. To show that $M_{i j}$ is unstable, we find $t_{i j} \in T_{i j}$ so that $\gamma\left(t_{i j}\right) \notin D\left(M_{i j}\right)$. In that case we say that the intersection of the wires $L_{i}$ and $L_{j}$ at $t_{i j}$ is unstable. This will depend on the sign of $i, j$ and/or the position of other curves $L_{k}, L_{\ell}$ on the critical line.

Lemma 6.2 Suppose $i<k<j$ and $t_{0} \in \mathbb{R}$ so that $f_{i}\left(t_{0}\right) \neq f_{j}\left(t_{0}\right)$. Suppose either

(1) $\varepsilon_{k}=+$ and $f_{k}\left(t_{0}\right) \leq \min \left(f_{i}\left(t_{0}\right), f_{j}\left(t_{0}\right)\right)$ or

(2) $\varepsilon_{k}=-$ and $f_{k}\left(t_{0}\right) \geq \max \left(f_{i}\left(t_{0}\right), f_{j}\left(t_{0}\right)\right)$.

Then $M_{i j}$ is unstable.

Proof In both cases there are two subcases: (a) $i \triangleleft j$ and (b) $j \triangleleft i$. We may assume $t_{i j}$ is unique since, otherwise, $M_{i j}$ is not stable. In Subcase (a), $t_{i j}>t_{0}$ and, in Subcase (b), $t_{i j}<t_{0}$. In all four subcases, $k$ is a witness to the instability of $M_{i j}$ (Definition 5.8). For example, in Case (1a), $f_{i}(t)=f_{k}(t)$ for some $t \leq t_{0}$. So, $t_{i k}^{0} \leq t_{0}<t_{i j}$ making $k$ a witness. In Case (1b), $f_{j}(t), f_{k}(t)$ switch order at some $t \geq t_{0}$. So $t_{k j}^{1} \geq t_{0}>t_{i j}$ making $k$ a witness. Cases (2a), (2b) are similar. Thus $M_{i j}$ is not stable.

Lemma 6.3 (Three-Point Lemma) Suppose there are three points on the critical line in one of the following two arrangements:

$\begin{array}{cccccc}j & . & & & k & - \\ i & . & & \text { or } & j & \\ k & + & & & i & \end{array}$

I.e., $k \triangleleft i \triangleleft j$ with $\varepsilon_{k}=+$ in the first case and $i \triangleleft j \triangleleft k$ with $\varepsilon_{k}=-$ in the second case. Then there is at most one stable intersection of the form $t_{i, j^{\prime}}$ where $k<i<k+n$ and $j=j+$ pn for some $p$, namely the one given by $k<i, j^{\prime}<k+n$.

Proof Case 1: If $j^{\prime}<k$ then $j^{\prime}<k<i$ making $M_{j^{\prime} i}$ unstable by Lemma 6.2 (1). Similarly, if $k+n<j^{\prime}$, then $i<k+n<j^{\prime}$ making $M_{i j^{\prime}}$ unstable. So, there is only one possible value of $j^{\prime}=j+p n$ for which $M_{i j^{\prime}}$ could be stable, namely the one with $k<j^{\prime}<k+n$.

Case 2 is similar using Lemma 6.2 (2).

Lemma 6.4 (Two-Point Lemma) Suppose there are two points on the critical line in one of the following two arrangements:

$$
\begin{array}{ccccc}
j & . & & j & - \\
i & + & \text { or } & i & .
\end{array}
$$


I.e., $i \triangleleft j$ and either $\varepsilon_{i}=+$ or $\varepsilon_{j}=-$. Then there are at most two stable intersections of the form $t_{i, j^{\prime}}$ with $j^{\prime}=j+$ pn given by taking the two values of $j^{\prime}$ so that $i-n<j^{\prime}<i+n$.

Proof Take the first case. If $j^{\prime}<i-n$ then apply Lemma 6.2 with $k=i-n$ to see that $M_{i j^{\prime}}$ is unstable. If $j^{\prime}>i+n$ we let $k=i+n$. Then, again, $M_{i j^{\prime}}$ is unstable by Lemma 6.2. So, the only possible stable $M_{i j^{\prime}}$ are the two with $i-n<j^{\prime}<i+n$.

The second case is similar.

Lemma 6.5 (Four-Point Lemma) Suppose there are four points on the critical line in the following arrangement:

$\begin{array}{cc}j & \cdot \\ \ell & - \\ k & + \\ i & .\end{array}$

I.e, $i \triangleleft k \triangleleft \ell \triangleleft j, \varepsilon_{k}=+$ and $\varepsilon_{\ell}=-$. Then there are at most two stable intersections of the form $t_{i, j+p n}$. Choosing $i, j, k, \ell$ so that $k<j, \ell<k+n$ and $\ell<i<\ell+n$, the possible stable intersections are $t_{i j}$ and $t_{i, j+n}$.

Proof This follows from Theorem 5.9 using the given ordering:

$$
k<\ell<i<\ell+n<k+2 n .
$$

For any $j^{\prime}=j+p n<k$ we have $t_{i, j^{\prime}}<c^{0}<t_{k \ell}^{1}$. So, $M_{i, j^{\prime}}$ is unstable by Theorem 5.9. Similarly, if $j^{\prime}>k+2 n$ we have $t_{\ell+n, k+2 n}^{0}<c^{0}<t_{i, j^{\prime}}$ making $M_{i, j^{\prime}}$ unstable. Thus the only possible values of $j^{\prime}=j+p n$ making stable $M_{i, j^{\prime}}$ are the two with $k<j^{\prime}<$ $k+2 n$.

Lemma 6.6 (Finiteness Lemma) Suppose that, on the critical line $t=c^{0}$, all positive points are above all negative points. I.e., $k \triangleright \ell$ whenever $\varepsilon_{k}=+$ and $\varepsilon_{\ell}=-$. Then there are an infinite number of stable modules.

Proof For any positive integer $p$ we consider the (finite) set of all pairs $(i, j)$ with $-p n \leq$ $i<j \leq p n$ so that $\varepsilon_{i}=+, \varepsilon_{j}=-$. Since $f_{i}\left(c^{0}\right)>f_{j}\left(c^{0}\right)$ the curves $L_{i}, L_{j}$ must cross at some point $t_{i j}<c^{0}$. Choose $(i, j)$ so that $t_{i j}<c^{0}$ is maximal and, if there is a tie, choose whichever has smaller value of $j-i$. Then we claim that this intersection is stable. To see this consider any $i<k<j$ with $\varepsilon_{k}=+$. If $f_{k}\left(t_{i j}\right) \leq f_{j}\left(t_{i j}\right)$ then $L_{k}, L_{j}$ cross at some point $t_{i j} \leq t_{k j}<c^{0}$. Since $j-k<j-i$ this contradicts the choice of $(i, j)$. Thus $f_{k}\left(t_{i j}\right)>f_{j}\left(t_{i j}\right)$. Similarly, $f_{\ell}\left(t_{i j}\right)<f_{i}\left(t_{i j}\right)=f_{j}\left(t_{i j}\right)$ for all $i<\ell<j$ with $\varepsilon_{\ell}=-$. So, $M_{i j}$ is stable by Theorem 5.4.

Now increase $p$ by 1 . Then $-(p+1) n \leq i-n<j+n \leq(p+1) n$. Since $t_{i j}<c^{0}$ we have:

$$
f_{i-n}\left(t_{i j}\right)<f_{i}\left(t_{i j}\right)=f_{j}\left(t_{i j}\right)<f_{j+n}\left(t_{i j}\right) .
$$

So, the wires $L_{i-n}$ and $L_{j+n}$ cross at some point $t_{i j}<t_{i-n, j+n}<c^{0}$. So, there exists another stable $M_{i^{\prime}, j^{\prime}}$ with $t_{i j}<t_{i^{\prime} j^{\prime}}<c^{0}$. Proceeding in this way, we get an infinite sequence of nonisomorphic stable modules.

Proposition 6.7 Let $M_{i j}$ be a stable module in a maximal green sequence for the quiver $\widetilde{A}_{a, b}^{\varepsilon}$. Suppose $\varepsilon_{i}=\varepsilon_{j}$. Then $j-i<n$. 
Proof We exclude first the case when $i<j$ are congruent modulo $n$. In that case, $f_{i}\left(c^{0}\right)=$ $f_{j}\left(c^{0}\right)$. By the Finiteness Lemma, there must be either a negative point $\ell \triangleleft i$ or a positive point $k \triangleright i$. By adding multiples of $n$ we can assume $i<k, \ell<j$. Then $M_{i j}$ is unstable by Theorem 5.4. In the remaining cases when $i<j$ are not congruent module $n$, by the 2-point Lemma 6.4 we must have $j-i<n$.

\subsection{The Upper Bound}

From the above lemmas, we can determine an upper bound for the number of stable intersections when finite: By the Finiteness Lemma there must be at least one positive point below a negative point. We will show that the following pattern of points on the critical line is the one which gives the largest finite number of stable intersections.

$$
\begin{aligned}
& a-1 \text { positive points }\left\{\begin{array}{l}
+ \\
\vdots \\
+
\end{array}\right.
\end{aligned}
$$

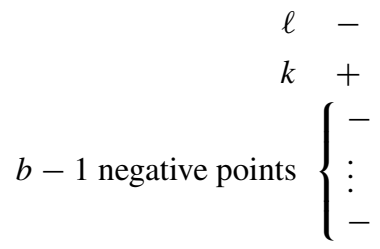

Theorem 6.8 Maximal green sequences for a quiver of type $\widetilde{A}_{a, b}$ have length at most

$$
\left(\begin{array}{c}
a+b \\
2
\end{array}\right)+a b .
$$

In every maximal green sequence of this length, the pattern of points on the critical line must be as given in Eq. 6.1 above.

Proof Considering the points on the critical line, let $f_{k}\left(c^{0}\right)$ be minimal among all points with $\varepsilon_{k}=+$ and let $f_{\ell}\left(c^{0}\right)$ be maximal for $\varepsilon_{\ell}=-$. By the Finiteness Lemma 6.6, $k \triangleleft \ell$. By adding a multiple of $n$ to $\ell$ if necessary, we may assume $k<\ell<k+n$.

Let $A$ denote the set of all $j$ with $k<j<k+n$ so that $k \triangleleft j$. Let $A^{\prime}$ be the subset of $A$ consisting of those $j$ with $\ell \unlhd j$. Then $A^{\prime}$ contains $\ell$ and the points above $\ell$ which are all positive. Since $k$ is positive and below $\ell, a^{\prime}=\left|A^{\prime}\right| \leq a$.

Similarly, let $B$ denote the set of all $i$ with $\ell-n<i<\ell$ so that $i \triangleleft \ell$ and let $B^{\prime}$ be the subset of $B$ consisting of those $i$ with $i \unlhd k$. Then $b^{\prime}=\left|B^{\prime}\right| \leq b$. On the critical line $t=c^{0}$, the set of points corresponding to $A$, resp $A^{\prime}$, is complementary to the set corresponding to $B^{\prime}, \operatorname{resp} B$. So, $|A|=n-b^{\prime}$ and $|B|=n-a^{\prime}$.

Consider all pairs $i, j$ (up to translation by $n$ ) so that $i \triangleleft j$. Since $f_{p}\left(c^{0}\right)$ takes $n$ values (given by $p=1,2, \cdots, n)$ there are $\left(\begin{array}{l}n \\ 2\end{array}\right)$ such pairs $i, j$.

Claim (a): If $i \in B^{\prime}$ and $j \in A^{\prime}$ there are at most 2 values of $p$ so that $M_{i, j+p n}$ is stable, namely $p=0,-1$ (i.e., only $M_{i j}$ and $M_{i, j-n}$ might be stable.)

Claim (b): If $i \notin B^{\prime}+n \mathbb{Z}$ or $j \notin A^{\prime}+n \mathbb{Z}$, there is at most one value of $p$ so that $M_{i, j+p n}$

is stable. 
In other words, the pair $(i, j)$ gives at most one stable module unless $i \in B^{\prime}$ and $j \in A^{\prime}$ in which case there might be two stable modules. So, the Claim implies that there are at most $\left(\begin{array}{l}n \\ 2\end{array}\right)+a^{\prime} b^{\prime}$ stable modules. This is maximal when $a^{\prime}=a$ and $b^{\prime}=b$ which happens exactly when the pattern of points on the critical line is given by Eq. 6.1. Thus, these claims imply the Theorem.

Proof of Claim (b): Suppose $i \notin B^{\prime}+n \mathbb{Z}$. Then $i, j \in A$ (up to translation by a multiple of $n$ ). By the 3-point Lemma (6.3), only $M_{i j}$ might be stable for $i, j \in A$. Similarly, if $j \notin A^{\prime}+n \mathbb{Z}$ then we may assume $i, j \in B$ in which case, again, only $M_{i j}$ might be stable.

Proof of Claim (a): Given $i \in B^{\prime}, j \in A^{\prime}$ we apply the 4-point Lemma 6.5 to $\ell<i+n<$ $\ell+n$ and $k<j<k+n$ to give the statement of Claim (a) in the case when $i \neq k$ and $j \neq \ell$. In the special cases when either $i=k$ or $j=\ell$, the statement of Claim (a) is give by the 2-point Lemma 6.4 .

This proves Claims (a) and (b). The Theorem follows.

Claims (a) and (b) in the above proof also imply the following converse of the Finiteness Lemma. Given a green path $\gamma$ with corresponding functions $f_{i}$ we say that $(k, \ell)$ is an essential pair for $\gamma$ if $\varepsilon_{k}=+, \varepsilon_{\ell}=-$ and $k \triangleleft \ell\left(f_{k}\left(c^{0}\right)<f_{\ell}\left(c^{0}\right)\right)$.

Theorem 6.9 (Finiteness Theorem) There are only finitely many $\gamma$ stable modules if and only if there exists an essential pair for $\gamma$

Proof This condition is necessary by the Finiteness Lemma 6.6. The converse follows from the proof of Theorem 6.8 above since Claims (a) and (b) in the proof imply that there are at most $\left(\begin{array}{l}n \\ 2\end{array}\right)+a^{\prime} b^{\prime}$ stable modules where $a^{\prime}=\left|A^{\prime}\right|, b^{\prime}=\left|B^{\prime}\right|$ are the sizes of the two finite sets in the proof of Theorem 6.8 which exist when there is an essential pair.

\subsection{Sets of Stable Modules}

Examining the details of the proof of Theorem 6.8, we obtain the list of stable modules of the maximum length:

Definition 6.10 For any $k<\ell<k+n$ with $\varepsilon_{k}=+$, $\varepsilon_{\ell}=-$, let $A_{k \ell}, B_{k \ell}$ denote the following sets of integers.

$$
\begin{aligned}
A_{k \ell} & :=\{\ell\} \cup\left\{j: k<j<k+n, \varepsilon_{j}=+\right\} \\
B_{k \ell} & :=\{k\} \cup\left\{i: \ell-n<i<\ell, \varepsilon_{i}=-\right\}
\end{aligned}
$$

Note that $\left|A_{k \ell}\right|=a,\left|B_{k \ell}\right|=b$. Let $\mathcal{S}_{k \ell}$ denote the set of modules in the following list.

(1) $M_{i j}$ where $i, j \in A_{k \ell}$ with $i<j$

(2) $M_{i j}$ where $i, j \in B_{k \ell}$ with $i<j$

(3) $M_{i j}$ (or $M_{j i}$ if $i>j$ ) where $j \in A_{k \ell}, i \in B_{k \ell}$

(4) $M_{i, j-n}\left(\right.$ or $M_{j-n, i}$ if $i>j-n$ ) where $j \in A_{k \ell}, i \in B_{k \ell}$

Theorem 6.11 For any maximal green sequence of length $\left(\begin{array}{c}a+b \\ 2\end{array}\right)+a b$, the set of stable modules is one of the sets $\mathcal{S}_{k \ell}$ defined above.

Proof The sets $A^{\prime}, B^{\prime}$ in the proof of Theorem 6.8 are equal to $A_{k \ell}, B_{k \ell}$ in the case when the pattern of points on the critical line is as given in Eq. 6.1. The possible stable modules of Claim (b) in the proof are listed in (1) and (2) in Definition 6.10. The possible stable modules of Claim (a) in the proof are listed in (3) and (4) in Definition 6.10. 
Lemma 6.12 If $(a, b) \neq(2,2)$, the sets $\mathcal{S}_{k \ell}$ are distinct.

Proof We will show that, if $a=1$ or $a \geq 3$, the sets $\mathcal{S}_{k \ell}$ are distinct, i.e., the set $\mathcal{S}=\mathcal{S}_{k \ell}$ determines $k$ and $\ell$ (modulo $n$ ). The case $b \neq 2$ is analogous.

Case 1: $\quad a=1$. Then $k$ is unique modulo $n$. Given $k$, the set $\mathcal{S}=\mathcal{S}_{k \ell}$ determines $\ell$ since $\ell$ is the only integer between $k$ and $k+n$ for which $M_{k \ell}$ and $M_{\ell-n, k}$ are both in the set $\mathcal{S}$. So, the $b$ sets $\mathcal{S}_{k \ell}$ are distinct.

Case 2: $\quad a \geq 3$. Then we claim that the set $\mathcal{S}=\mathcal{S}_{k \ell}$ uniquely determines $\ell$ modulo $n$. The reason is that $\ell$ is the unique integer modulo $n$ with $\varepsilon_{\ell}=-$ which is "doubly paired" with only one integer $k$ modulo $n$ with $\operatorname{sign} \varepsilon_{k}=+$ where, by "doubly paired", we mean there are two values of $p$ for which $M_{\ell, k+p n}$ are in $\mathcal{S}$. (The negative points not equal to $\ell$ are paired with the $a-1 \geq 2$ positive points not equal to $k$.) Furthermore, $\ell$ determines $k$ modulo $n$ since $k$ and $\ell$ are doubly paired.

In the next section we will show (Theorem 7.10) that each of the sets $\mathcal{S}_{k \ell}$ occur as the stable set of modules of some maximal green sequence for $\widetilde{A}_{a, b}^{\varepsilon}$ for any sign function $\varepsilon$. Together with the above lemma, this implies the following

Theorem 6.13 (Theorem M2) For $(a, b) \neq(2,2)$ there are exactly ab distinct sets of stable

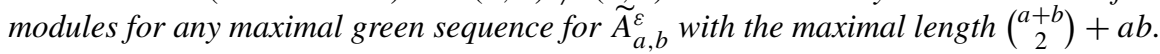

The case $(a, b)=(2,2)$ is an easy exercise:

Proposition 6.14 For the quivers $\widetilde{A}_{2,2}^{+-+-}$, resp. $\widetilde{A}_{2,2}^{++-}$, there are 2 , resp. 3, possible sets of stable modules for the maximal green sequences of the maximum length which is 10.

Proof For $\tilde{A}_{2,2}^{+-+-}$, the possible values of the essential pair $(k, \ell)$ are $(1,2),(1,4),(3,4),(3,6)$. But $A_{14}=\{3,4\}=B_{36}$ and both elements of $A_{36}=\{5,6\}$ are $n=4$ more than the elements of $B_{14}=\{1,2\}$. So, $\mathcal{S}_{14}=\mathcal{S}_{36}$. Similarly, $\mathcal{S}_{12}=\mathcal{S}_{34}$. So, there are only two distinct sets of the form $\mathcal{S}_{k \ell}$.

For $\widetilde{A}_{2,2}^{++-}$, the formula for $\mathcal{S}_{k \ell}$ gives:

\begin{tabular}{c|cc|c}
$(k, \ell)$ & $B_{k \ell}$ & $A_{k \ell}$ & $i j$ with $M_{i j} \in \mathcal{S}_{k \ell}$ \\
\hline$(1,3)$ & $\{1,0\}$ & $\{3,2\}$ & $01,23,02,03,12,13,24,25,34,35=\mathcal{S}_{24}$ \\
$(1,4)$ & $\{1,3\}$ & $\{4,2\}$ & $24,13,12,14,23,34,25,27,01,03$ \\
$(2,3)$ & $\{2,0\}$ & $\{3,5\}$ & $02,35,03,05,23,25,34,36,01,12$ \\
$(2,4)$ & $\{2,3\}$ & $\{4,5\}$ & $23,01,24,25,34,35,02,03,12,13=\mathcal{S}_{13}$
\end{tabular}

Thus $\mathcal{S}_{14} \neq \mathcal{S}_{23}$ with neither equal to $\mathcal{S}_{13}=\mathcal{S}_{24}$. So, there are three distinct sets $\mathcal{S}_{k \ell}$.

\subsection{Deletion Lemma}

One immediate consequence of Theorem 5.4 and Corollary 5.6, two theorems we had earlier, is the observation that, when a curve $L_{k}$ is deleted, stable intersections of other curves remain stable. However, previously unstable intersections, possibly an infinite number of them, might become stable. Using Theorem 6.9 we can prevent this from happening.

We need some notation. For $Q$ a quiver of type $\widetilde{A}_{a, b}$ and $X$ a subset of the arrow set of $Q$ having at most $a+b-2$ elements, we say that $Q^{\prime}$ is obtained by collapsing the arrows in 
$X$ if $Q^{\prime}$ is given by deleting each arrow $\alpha_{i}$ (from $i$ to $i+1$ or $i+1$ to $i$ ) in $X$ and identifying the source and target of $\alpha_{i}$. This induces an epimorphism

$$
\bar{\pi}: Q_{0}=\{1,2, \cdots, n\} \rightarrow Q_{0}^{\prime}=\{1,2, \cdots, n-|X|\}
$$

which is the reduction modulo $n-|X|$ of a map $\pi: \mathbb{Z} \rightarrow \mathbb{Z}$ which is periodic in the sense that $\pi(i+n)=\pi(i)+n-|X|$ and so that $\pi(1)=1, \pi(i) \leq \pi(j)$ when $i \leq j$ and $\pi(i)=\pi(i+1)$ when $\alpha_{i} \in X$. Given a module $M_{i j}$ for $K Q$, let $\bar{\pi}\left(M_{i j}\right)$ be the $K Q^{\prime}$ module given by

$$
\bar{\pi}\left(M_{i j}\right)= \begin{cases}0 & \text { if } X \text { contains } i \text { or } j \\ M_{\pi(i) \pi(j)} & \text { otherwise }\end{cases}
$$

Proposition 6.15 (Deletion Lemma) Let $M_{1}, \cdots, M_{m}$ be a maximal green sequence for $\Lambda=K Q$ for $Q$ a quiver of type $\widetilde{A}_{a, b}$. Let $\gamma$ be a corresponding green path. Let $Q^{\prime}$ be $a$ quiver obtained from $Q$ by collapsing a set of arrows $X$ in $Q$ where $X$ is disjoint from at least one essential pair for $\gamma$. Then there is a maximal green sequence for $K Q^{\prime}$ containing as a subsequence the nonzero elements of the sequence $\bar{\pi}\left(M_{1}\right), \cdots, \bar{\pi}\left(M_{m}\right)$. Furthermore, if the first sequence is linear, so is the second.

Remark 6.16 It is an easy exercise to show that collapsing arrows in $X$ sends $\mathcal{S}_{k \ell}$ to $\mathcal{S}_{k \ell}$ (a different set with the same name) as long as $X$ is disjoint from the essential pair $(k, \ell)$. This lemma does not follow from [21] since we are deleting arrows, not vertices.

\subsection{Cluster-Tilted $D_{n}$}

The analysis above also applies to the case when $b=0$. Then the quiver is a single oriented cycle of length $n=a$. Call this quiver $Q_{n}$. We impose the condition $\operatorname{rad}^{n-1}=0$, i.e., the composition of any $n-1$ arrows is zero. The path algebra $K Q_{n}$ modulo $\mathrm{rad}^{n-1}$ is the "Jacobian algebra" of a quiver with potential $\Lambda_{n}=J\left(Q_{n}, W\right)$. This is "cluster-tilted" of type $D_{n}$. For more details, please see the paper [17] which was written to explain clustertilted algebras of Dynkin type and the problem of finding a maximal green sequence for these algebras.

The algebra $\Lambda_{n}$ has, up to isomorphism, $n(n-1)$ indecomposable modules $M_{i j}$ where $1 \leq i \leq n$ and $i<j<i+n$. Given any green path $\gamma(t)=t \mathbf{b}(t)-\mathbf{a}(t)$ for $\Lambda_{n}$ we have associated functions $f_{i}: \mathbb{R} \rightarrow \mathbb{R}^{n}$ given, just as in the case of $\widetilde{A}_{a, b}$, by Eq. 5.3. The critical line $t=c^{0}$ is defined as before. The Finiteness Lemma does not hold since there are only finitely many stable modules. However Proposition 6.7 holds by definition and the 2-, 3and 4- point lemmas all hold with the same proofs as before.

Maximal green sequences of maximal length for $\Lambda_{n}$ will arise from the collapsing process described in the Deletion Lemma 6.15 using the following observation.

Remark 6.17 Let $\Lambda=K Q$ be the algebra of type $\widetilde{A}_{n, 1}^{\varepsilon}$ with sign function $\varepsilon_{i}=-$ if and only if $i$ is a multiple of $n+1$. Then $Q_{n}$ is obtained from $Q$ by collapsing the unique edge in $Q$ with negative sign. The collapsing map $\pi$ from the Deletion Lemma sends the set of $\Lambda$-modules $\mathcal{S}_{k, n+1}$ to the set of $\Lambda_{n}$-modules

$$
\mathcal{S}_{k}:=\left\{M_{i j}: k \leq i<j \leq k+n, j-i<n\right\}
$$

which has $\left(\begin{array}{l}n \\ 2\end{array}\right)+n-1$ elements. We observe that the $n$ sets $\mathcal{S}_{k}$ are distinct. 
Theorem 6.18 Maximal green sequences for $\Lambda_{n}=K Q_{n} / \mathrm{rad}^{n-1}$ have length at most

$$
\left(\begin{array}{l}
n \\
2
\end{array}\right)+n-1
$$

and each such maximal green sequence has stable set of modules $\mathcal{S}_{k}$ for some $k$.

Proof Let $f_{k}\left(c^{0}\right)$ be the lowest point on the critical line. By the 2-point Lemma 6.4 and the 3-point Lemma 6.3, the only possible stable modules are the elements of $\mathcal{S}_{k}$.

We give a central charge $Z: K_{0} \Lambda_{n} \rightarrow \mathbb{C}$ which realizes each maximal stability set $\mathcal{S}_{k}$. Together with Theorem 6.18 above, this will prove Corollary L4 from the introduction. (See Fig. 14 for the corresponding chord diagram.)

Corollary 6.19 For every $k$ there is a standard linear stability condition on $\Lambda_{n}$ with stable set of modules $\mathcal{S}_{k}$.

Proof Take $\mathbf{b}=(1,1, \cdots, 1)$ and let $\mathbf{a}=\left(a_{1}, \cdots, a_{n}\right)$ be given by $a_{j}=c_{j}-c_{j-1}$ where $c_{j}=-(2 k+n-2 j)^{2}$.

\section{Chord Diagrams for $\tilde{A}_{a, b}$}

In this section we use (periodic) chord diagrams to construct linear stability conditions having the maximum number of stable modules. We also construct piecewise linear green paths having any given maximal set $\mathcal{S}_{k \ell}$ of stable modules as given in Definition 6.10 completing the proof of Theorem M2.

\subsection{Periodic Stablility Polygon $\tilde{C}(Z)$}

The first step is to show that, without loss of generality, we may assume that $m=0$ and $c$, the critical slope of $Z$, is also zero. We have already noted that the value of $m$ is irrelevant. Setting $m=0$ makes:

$$
f_{i}(t)=a_{1}+\cdots+a_{i}-t\left(b_{1}+\cdots+b_{i}\right)
$$

We translate all wires $L_{i}$ to the left by $c$ units by replacing $f_{i}$ with

$$
f_{i}^{c}(t)=f_{i}(t+c)=\left(a_{1}-c b_{1}\right)+\cdots+\left(a_{i}-c b_{i}\right)-t\left(b_{1}+\cdots+b_{i}\right) .
$$

This corresponds to a new central charge $Z^{c}$ given by $Z^{c}(x)=(\mathbf{a}-c \mathbf{b}) \cdot x+i \mathbf{b} \cdot x$. Then $Z^{c}$ has the same stable modules as does $Z$ with the slopes of all modules decreased by $c$. Thus, without loss of generality, we may replace $Z$ with $Z^{c}$ and assume that $c=0$. Equivalently,

$$
a_{1}+\cdots+a_{n}=0 .
$$

We say that $Z$ is normalized if it has these two properties $(m=c=0)$.

As in the finite $A_{n}$ case we define the periodic dual vertices $p_{i} \in \mathbb{R}^{2}$ for $i \in \mathbb{N}$ by

$$
p_{i}=\left(b_{1}+\cdots+b_{i}, a_{1}+\cdots+a_{i}\right)
$$

Since $c=0, p_{i+n}=p_{i}+(B, 0)$ for all $i \in \mathbb{N}$ where $B=b_{1}+\cdots+b_{n}$. For $i<0$ we can then define $p_{i}=p_{i+k n}-k(B, 0)$ for sufficiently large $k$.

For all integers $i<j$ the chord $V_{i j}$ is the line segment with endpoints $p_{i}, p_{j}$. As in the finite case (Theorem 1.5) we have the following. 
Theorem 7.1 For $Z$ a normalized central charge on $K \widetilde{A}_{a b}^{\varepsilon}$, a module $M_{i j}$ is $Z$-semistable if and only if the chord $V_{i j}$ has no negative dual vertices above it and no positive vertices below it. $M_{i j}$ is $Z$-stable if, in addition, $V_{i j}$ has no dual vertices in its interior.

For example, $M_{i, i+2 n}$ is never $Z$-stable since $p_{i+n}$ is in the interior of $V_{i, i+2 n}$.

In the following corollary it is essential that $Z$ be normalized. Then $p_{i}, p_{i+n}$ have the same height $y_{i}=y_{i+n}$ for all $i \in \mathbb{Z}$. So, at most $n$ distinct heights are attained.

Corollary 7.2 If the height $y_{k}$ of every positive dual vertex $p_{k}$ is greater than the height $y_{\ell}$ of every negative dual vertex $p_{\ell}$ then there are infinitely many nonisomorphic Z-stable modules $M_{i j}$.

Proof Let $p_{k}$ be a positive dual vertex with minimum height $y_{k}$. Let $p_{\ell}$ be a negative dual vertex of maximum height $y_{\ell}$. Then $y_{k}>y_{\ell}=y_{\ell+s n}$ for all integers $s$. Then any interior point in any chord of the form $V_{k, \ell+s n}$ has $y$-coordinate greater than $y_{\ell}$ and less than $y_{k}$. By Theorem 6.1 the corresponding modules $M_{k, \ell+s n}$ are $Z$-stable for all integers $s$.

In analogy with the finite case, the periodic stability polygon $\widetilde{C}(Z)$ is defined to be the intersection

$$
\widetilde{C}(Z)=\widetilde{C}^{+}(Z) \cap \widetilde{C}^{-}(Z)
$$

where $\widetilde{C}^{+}(Z), \widetilde{C}^{-}(Z)$ are defined as follows. Let $p_{k_{i}}$ be the positive dual vertices with fixed indexing $k_{i}, i \in \mathbb{Z}$. For every pair of consecutive elements in this set, $p_{k_{i-1}}, p_{k_{i}}$, let $\widetilde{C}_{i}^{+}(Z)$ be the union of the convex hull of the points $p_{j}$ for $k_{i-1} \leq j \leq k_{i}$ with the set of all points below this convex hull, i.e., points with $x$-coordinate equal and $y$-coordinate less than a point in this convex hull. Define $\widetilde{C}^{+}(Z)$ to be the union of these sets $\widetilde{C}_{i}^{+}(Z)$. Similarly, $\widetilde{C}^{-}(Z)$ is the union of sets $\widetilde{C}_{j}^{-}(Z)$ which are defined to be the convex hull of all dual vertices $p_{i}$ between and including two consecutive negative vertices union the set of all points above this convex hull.

Theorem 6.1 is equivalent to the following affine analogue of Theorem 1.8.

Theorem 7.3 For a normalized central charge $Z$ on $K \widetilde{A}_{a b}^{\varepsilon}$, the string module $M_{i j}$ is $Z$ semistable if and only if $V_{i j} \subset \widetilde{C}(Z) . M_{i j}$ is $Z$-stable if, in addition, the chord $V_{i j}$ contains no dual vertex $p_{k}$ in its interior.

\subsection{Linearity of Maximal Stable Sets}

Theorem 7.4 Suppose $k<\ell<k+n$. Then, a sufficient condition for the stable set $\mathcal{S}_{k \ell}$ to be linear is if, in the sequence of signs $\varepsilon_{j}$ for $k<j<\ell$, all negative signs come before all positive signs. Another sufficient condition is if, in the sequence of signs $\varepsilon_{j}$ for $\ell<j<k+n$, all positive signs come before all negative signs.

Remark 7.5 Theorem 7.6 below shows that if neither of these conditions holds, the stable set $\mathcal{S}_{k \ell}$ is nonlinear. Thus, these conditions, taken together, are necessary and sufficient.

Proof We prove the first statement. The second is analogous. The proof is given by drawing one periodic stability polygon (Fig. 11) which will generate all examples.

The points $p_{1}, \cdots, p_{6}$ have coordinates $(-1,-2),(0,0),(2,2),(4, \varepsilon-2),(6, \varepsilon),(7,2+$ $\varepsilon),(12,-2)$, etc with $p_{i+6}=p_{i}+(13,0)$. Then $V_{12}, V_{45}$ have slope $2, V_{23}, V_{45}$ have slope 
$1, V_{24}, V_{35}$ have slope approximately $-1 / 2$ and $V_{57}, V_{68}$ have slope approximately $-1 / 3$. In the drawing $\varepsilon=0$. But any positive $\varepsilon<2 / 5$ will make all 24 chords of $\mathcal{S}_{25}$ stable.

To draw more general cases, we should insert vertices along the chords $V_{14}$ and $V_{36}$ along a curve (in green in Fig. 11) which is slightly concave up along $V_{14}$ and slightly concave down along $V_{36}$. As long as all the positive points between $\ell$ and $k+n$ come before all the negative points, such a figure will be accurate and make all chords in $\mathcal{S}_{k \ell}$ stable.

Theorem 7.6 The stable set of modules $\mathcal{S}_{k \ell}$ is nonlinear if and only if there exist $k<k^{\prime}<$ $\ell^{\prime}<\ell<\ell^{\prime \prime}<k^{\prime \prime}<k+n$ so that $\varepsilon_{k^{\prime}}, \varepsilon_{k^{\prime \prime}}$ are positive and $\varepsilon_{\ell^{\prime}}, \varepsilon_{\ell^{\prime \prime}}$ are negative.

Proof If $k^{\prime}, k^{\prime \prime}, \ell^{\prime}, \ell^{\prime \prime}$ do not all exist then the stable set $\mathcal{S}_{k \ell}$ is linear by Theorem 6.4. Therefore, it suffices to show that, when they do exist, $\mathcal{S}_{k \ell}$ is nonlinear.

By the Deletion Lemma 5.15, we may delete (collapse) all the other edges of the quiver and assume that the quiver is $\widetilde{A}_{33}^{+++---}$with $k=2, \ell=5$. Consider the periodic stability polygon $\widetilde{C}(Z)$ with vertices $p_{i}$. If we write $p_{i}=\left(x_{i}, y_{i}\right)$ then

$$
y_{i}=y_{i+6 m}=a_{1}+\cdots+a_{i}=f_{i}(0) .
$$

By construction of the maximal stability set $\mathcal{S}_{k \ell}=\mathcal{S}_{25}$ we therefore have:

$$
y_{0}, y_{4}<y_{2}<y_{5}<y_{1}, y_{3}
$$

But $\mathcal{S}_{25}$ contains $M_{03}, M_{14}, M_{36}, M_{47}$. Since these are stable, the dual vertex $p_{2}$ must be above the chords $V_{03}, V_{14}$. So, $y_{2}$ must be greater than the $y$-coordinate of $\left(s_{1}, s_{2}\right)=V_{03} \cap$ $V_{14}$. Similarly, the $y$-coordinate of $\left(t_{1}, t_{2}\right)=V_{36} \cap V_{47}$ must be greater than $y_{5}$. So,

$$
s_{2}<y_{2}<y_{5}<t_{2} .
$$

However, examination of Fig. 12 shows that $t_{2}<s_{2}$ which gives a contradiction. The proof is: move $p_{1}$ (and $p_{7}$ ) left until it is right above $p_{0}$ (and $p_{7}$ above $p_{6}$ ). Then move $p_{3}=\left(x_{3}, y_{3}\right)$ right until $x_{3}=x_{4}$. Both moves decrease $s_{2}$ and increase $t_{2}$. After the moves we have $s_{2}=t_{2}$. So, the original values must be related by $s_{2}>t_{2}$. This is a contradiction showing that $\mathcal{S}_{25}$ is not the stability set corresponding to the height pattern Eq. 7.1. But the analysis of the critical line shows that this height pattern is the only one which can give this stability set. Therefore $\mathcal{S}_{25}$ is not the stability set of any central charge.

Corollary 7.7 If either a or $b$ is $\leq 2$, all maximal stable sets of modules are linear.

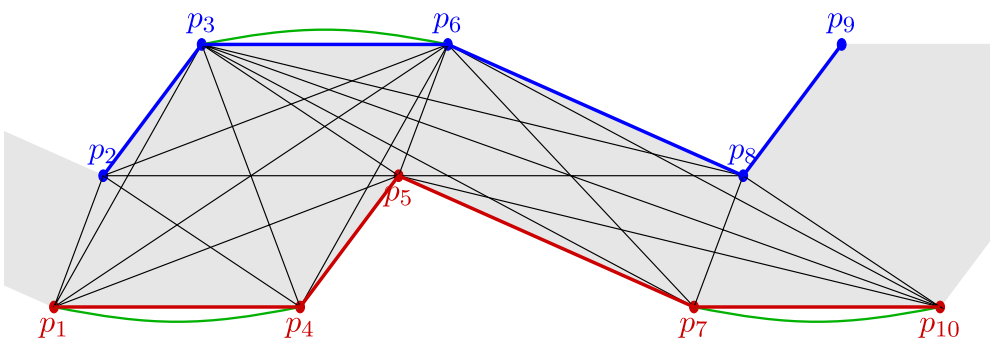

Fig. 11 This is a periodic chord diagram for $\widetilde{A}_{33}^{-++--+}$with all 24 chords of $\mathcal{S}_{25}$ being within the polygon and thus stable. Additional vertices can be placed along the green curves 


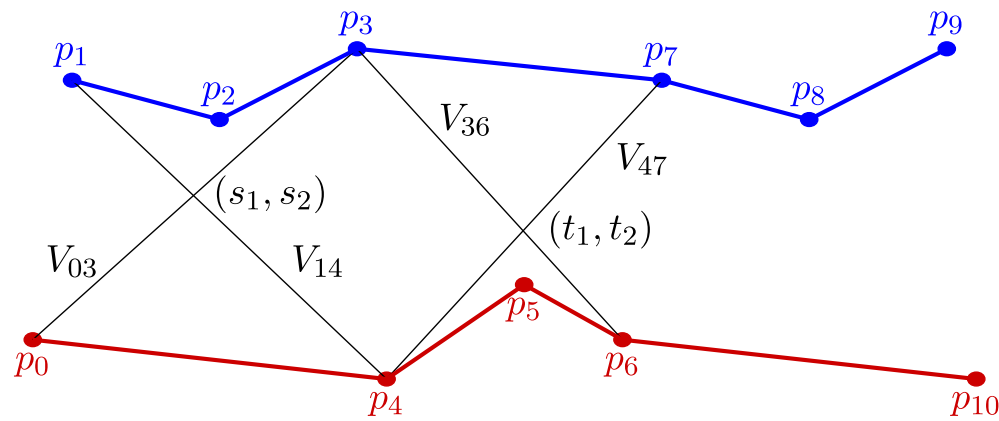

Fig. 12 When $M_{03}, M_{14}$ are stable, $p_{2}$ is higher than $\left(s_{1}, s_{2}\right)=V_{03} \cap V_{14}$. When $M_{36}, M_{47}$ are stable, $p_{5}$ is lower than $\left(t_{1}, t_{2}\right)=V_{36} \cap V_{47}$. But $t_{2}<s_{2}$, contradicting the assumption that $p_{2}$ is lower than $p_{5}$

\subsection{Nonlinear Maximal Stable Sets}

Let $\mathcal{S}_{k \ell}$ be one of the maximal stability sets identified as being nonlinear in Theorem 7.6. We will construct a piecewise linear green path $\gamma$ which crosses the walls $D(\beta)$ for $\beta \in \mathcal{S}_{k \ell}$ and no other walls. This green path will be formed from two linear green paths $\lambda, \lambda^{\prime}$ with the same value at $t=0: \lambda(0)=\lambda^{\prime}(0)$ by

$$
\gamma(t)= \begin{cases}\lambda(t) & \text { if } t \leq 0 \\ \lambda^{\prime}(t) & \text { if } t>0\end{cases}
$$

We say that $\gamma$ is given by splicing together $\lambda$ and $\lambda^{\prime}$ at $t=0$.

Since linear green paths are given by $\lambda(t)=t \mathbf{b}-\mathbf{a}$, two linear green paths take the same value at $t=0$ if and only if they have the same vector $\mathbf{a} \in \mathbb{R}^{n}$.

Lemma 7.8 Let $\gamma$ be given by splicing together linear green paths $\lambda_{Z}, \lambda_{Z^{\prime}}$ for stability functions $Z, Z^{\prime}$ with the same vector $a$. Suppose that $Z, Z^{\prime}$ have no semi-stable modules of slope 0 . Then the set of $\gamma$-stable, resp. $\gamma$-semistable, modules is the union of the following two sets.

(1) The set of $Z$-stable, resp. Z-semistable, modules $M$ with negative slope.

(2) The set of $Z^{\prime}$-stable, resp. $Z^{\prime}$-semistable, modules $N$ with positive slope.

Remark 7.9 Having the same vector $a$ means that, in the periodic chord diagrams $\widetilde{C}(Z), \widetilde{C}\left(Z^{\prime}\right)$, the corresponding dual vertices $p_{i}, p_{i}^{\prime}$ have the same $y$-coordinates. The stable/semistable chords of $\gamma$ are the stable/semistable chords of $\widetilde{C}(Z)$ of negative slope and the stable/semistable chords of $\widetilde{C}\left(Z^{\prime}\right)$ of positive slope.

Theorem 7.10 Given $\Lambda=K \widetilde{A}_{a b}^{\varepsilon}$ and $k<\ell<k+n$ with $\varepsilon_{k}=+, \varepsilon_{\ell}=-$ there exists a piecewise linear green path $\gamma$, given by splicing together two linear green paths as described above, so that all $\gamma$-semistable modules are stable and the set of $\gamma$-stable modules is $\mathcal{S}_{k}$ of We will construct the periodic stability polygons $\widetilde{C}(Z), \widetilde{C}\left(Z^{\prime}\right)$ having the required properties. The functions $Z, Z^{\prime}$ are determined by the coordinates of the dual vertices $p_{i}, p_{i}^{\prime}$ of $\widetilde{C}(Z), \widetilde{C}\left(Z^{\prime}\right)$, resp. As required, $p_{k}, p_{\ell}$ will have $y$-coodinates $y_{k}<y_{\ell}$. Then all modules of length $\geq 2 n$ will be unstable. So, the set of stable objects is finite and cannot be it greater than $\mathcal{S}_{k \ell}$. We assume that $p_{k-1}, p_{k+1}$ are positive and $p_{\ell-1}, p_{\ell+1}$ are negative. There is no 
loss of generality since, by the Deletion Lemma 6.15, we can delete these dual vertices and the remaining objects in $\mathcal{S}_{k \ell}$ will remain stable and give the set $\mathcal{S}_{k \ell}$ for the smaller quiver.

The dual vertices of $\widetilde{C}(Z)$ are given as follows (See Fig. 13):

(1) $p_{k}=(-14,-1)$ and $p_{k+n}=(26,-1)$. Thus $p_{i+n}=p_{i}+(40,0)$ for all $i \in \mathbb{Z}$.

(2) $p_{\ell}=(-5,1)$ and $p_{\ell+n}=(35,1)$.

(3) All positive dual vertices $p_{i}$ for $k<i<k+n$ lie on a parabolic curve from $p_{k+1}=$ $(-10,21)$ to $p_{k+n-1}=(11,19)$ which is concave down with slope between 0 and -1 at all points. Also, the $x$-coordinates of these $p_{i}$ lie in $[-10,-9] \cup[10,11]$.

(4) All negative dual vertices $p_{j}$ for $\ell<j<\ell+n$ lie on a parabolic curve from $p_{\ell+1}=$ $(10,-19)$ to $p_{\ell+n-1}=(31,-21)$ which is concave up with slope between 0 and -1 at all points. Also, the $x$-coordinates of these $p_{j}$ lie in $[10,11] \cup[30,31]$.

Let $A, B$ be the sets of positive and negative dual vertices listed in (3),(4) above. The parallel chords $V_{\ell, \ell+1}$ and $V_{k+n-1, k+n}$ have slope $-4 / 3$ which is $<-1$ and greater than the slopes of $V_{k+1, \ell}, V_{k+n, \ell+n-1}$ which are both -4 . Therefore, all chords between any two elements of the set $A \cup B \cup\left\{p_{\ell}, p_{k+n}\right\}$ are $Z$-stable. These lie in the shaded region of the left hand diagram in Fig. 13. All chords of $\mathcal{S}_{k \ell}$ of negative slope lie either in this set or have the form $V_{i j}$ where $k<i<j<\ell, \varepsilon_{i}=+, \varepsilon_{j}=-$. These lie in the yellow strip in the left side of Fig. 13. Thus, these are also $Z$-stable. Thus, all chords in $\mathcal{S}_{k \ell}$ with negative slope are $Z$-stable.

The dual vertices of $\widetilde{C}\left(Z^{\prime}\right)$ are the same as those of $\widetilde{C}\left(Z^{\prime}\right)$ except that $p_{k}$ moves 10 units left and $p_{\ell}$ moves 10 units right (so $p_{k}^{\prime}=(-24,-1)$ and $p_{\ell}^{\prime}=(5,1)$ ). Let $B^{\prime}$ be the set of negative dual vertices $p_{j}^{\prime}=p_{j}$ with $\ell-n<j<\ell$. Then, all chords between any two points in $A \cup B^{\prime} \cup\left\{p_{k}, p_{\ell}\right\}$ lie in the convex shaded region in $\widetilde{C}\left(Z^{\prime}\right)$ in Fig. 13. Together with the positive sloped chords $V_{i j}$ for $\ell<i<j<k+n$ with $\varepsilon_{i}=-, \varepsilon_{j}=+$, these are all the positive sloped chords in $\mathcal{S}_{k \ell}$.

Finally, note that no stable chords of $Z$ or $Z^{\prime}$ are horizontal. By Lemma 7.8, the green path obtained by splicing $\lambda_{Z}$ and $\lambda_{Z^{\prime}}$ makes all elements of $\mathcal{S}_{k \ell}$ stable.

Combining Theorems $6.11,7.10$ we get the following.
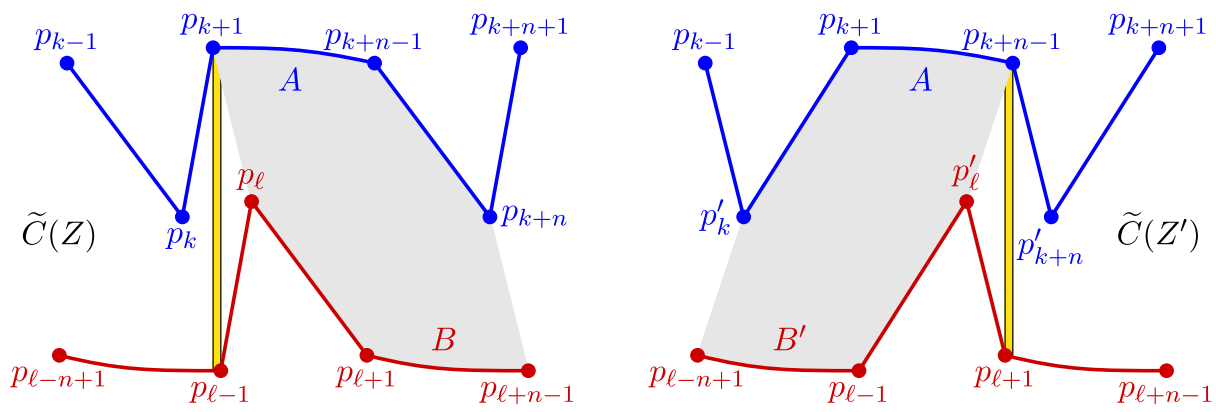

Fig. 13 Since each of the shaded (gray) regions is convex, the $\left(\begin{array}{l}n \\ 2\end{array}\right)$ chords in each shaded region are stable. All chords of negative slope in $\mathcal{S}_{k \ell}$ are in $\widetilde{C}(Z)$ either in the shaded region or in the yellow strip, both convex. Similarly, all chords in $\mathcal{S}_{k \ell}$ of positive slope are in the shaded region or yellow strip in $\widetilde{C}\left(Z^{\prime}\right)$. "Splicing" these makes all chords in $\mathcal{S}_{k \ell}$ stable 
Fig. 14 The $\left(\begin{array}{l}n \\ 2\end{array}\right)+n-1=14$ chords $V_{i j}$ for $1 \leq i<j \leq 6$ are stable

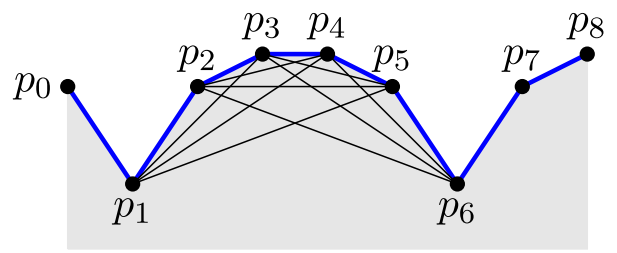

Corollary 7.11 Given $\Lambda=K \widetilde{A}_{a b}^{\varepsilon}$ and $k<\ell<k+n$ with $\varepsilon_{k}=+, \varepsilon_{\ell}=-$ there exists a maximal green sequence with stable module set equal to $\mathcal{S}_{k \ell}$. This set has $\left(\begin{array}{c}a+b \\ 2\end{array}\right)+a b$ elements. These are all the sets of stable modules of a maximal green sequence of this length and there are no maximal green sequences of greater length.

For the cluster-tilted algebra $\Lambda_{n}=J\left(Q_{n}, W\right)=K Q_{n} / \mathrm{rad}^{n-1}$, the linear maximal green sequence of Corollary 6.19 is illustrated in Fig. 14 in the case $n=5, k=1$.

Open Access This article is distributed under the terms of the Creative Commons Attribution 4.0 International License (http://creativecommons.org/licenses/by/4.0/), which permits unrestricted use, distribution, and reproduction in any medium, provided you give appropriate credit to the original author(s) and the source, provide a link to the Creative Commons license, and indicate if changes were made.

\section{References}

1. Assem, I., Simson, D., Skowroński, A.: Elements of the representation theory of associative algebras, 1: Techniques of representation theory London Mathematical Society Student Texts, vol. 65. Cambridge University Press, Cambridge (2006)

2. Auslander, M., Reiten, I., Smalø, S.O.: Representation theory of Artin algebras, vol. 36. Cambridge University Press, Cambridge (1997)

3. Bridgeland, T.: Stability conditions on triangulated categories. Ann. Math. 166(2), 317-345 (2007)

4. Bridgeland, T.: Spaces of stability conditions. Algebraic geometry-Seattle 1(2009), 1-21 (2005)

5. Brüstle, T., Dupont, G., Pérotin, M.: On maximal green sequences. Int. Math. Res. Not. 2014, 4547-4586 (2014)

6. Brüstle, T., Smith, D., Treffinger, H.: Stability conditions, tau-tilting theory and maximal green sequences. arXiv:1705.08227 (2017)

7. Buan, A.B., Marsh, R.J., Reineke, M., Reiten, I., Todorov, G.: Tilting theory and cluster combinatorics. Adv. Math. 204(2), 572-618 (2006)

8. Buan, A.B., Marsh, R.J., Reiten, I.: Cluster-tilted algebras. Trans. Amer. Math. Soc. 359(1), 323-332 (2007). (electronic)

9. Bucher, E.: Cluster algebras and maximal green sequences for closed surfaces (2016)

10. Chávez, A.N.: On the $c$-vectors of an acyclic cluster algebra. Int. Math. Res. Not. 6(2013), 1590-1600 (2015)

11. Derksen, H., Weyman, J.: Semi-invariants of quivers and saturation for Littlewood-Richardson coefficients. J. Amer. Math. Soc. 13(3), 467-479 (2000). (electronic)

12. Dlab, V., Ringel, C.M.: Indecomposable representations of graphs and algebras. Vol. 173 American Mathematical Soc. (1976)

13. Keller, B.: On cluster theory and quantum dilogarithm identities. arXiv: 1102.4148

14. Keller, B.: A survey on maximal green sequences, with an appendix by Laurent Demonet. arXiv: 1904.09247

15. Hermes, S., Igusa, K.: The no gap conjecture for tame hereditary algebras. Journal of Pure and Applied Algebra 223(3), 1040-1053 (2019)

16. Igusa, K.: Linearity of stability conditions. arXiv:1706.06986 (2017)

17. Igusa, K.: Maximal green sequences for cluster-tilted algebras of finite type. arXiv:1706.06503 (2017)

18. Igusa, K., Orr, K., Todorov, G., Weyman, J.: Modulated semi-invariants. arXiv:1507.03051v2

19. Kase, R.: Remarks on lengths of maximal green sequences for quivers of type $\widetilde{A}_{n, 1}$. arXiv: 1507.02852 
20. King, A.D.: Moduli of representations of finite dimensional algebras, Quart. J. Math. Oxford 45, 515530 (1994)

21. Muller, G.: The existence of a maximal green sequence is not invariant under quiver mutation. The Electronic Journal of Combinatorics 23(2), 2-47 (2016)

22. Qin, F.: Bases for upper cluster algebras and tropical points. arXiv:1902.09507

23. Qiu, Y.: Stability conditions and quantum dilogarithm identities for Dynkin quivers. Adv Math. 269, 220-264 (2015)

24. Reineke, M.: The Harder-Narasimhan system in quantum groups and cohomology of quiver moduli. Invent. Math. 152(2), 349-368 (2003)

25. Cormier, E., Dillery, P., Resh, J., Serhiyenko, K., Whelan, J.: Minimal length maximal green sequences and triangulations of polygons. J. Algebraic Combin. 44(4), 905-930 (2016)

26. Rudakov, A.: Stability for an abelian category. J. of Algebra 197, 231-245 (1997)

27. Yakimov, M.: Maximal green sequences for double Bruhat cells, preprint (2014)

Publisher's Note Springer Nature remains neutral with regard to jurisdictional claims in published maps and institutional affiliations. 